\title{
MAFIC DIKE SWARMS OF QUADRILÁTERO FERRÍFERO AND SOUTHERN ESPINHAÇO, MINAS GERAIS, BRAZIL
}

\author{
ADALENE MOREIRA SILVA , FARID CHEMALE $\mathbf{J R}^{* *}$, \\ RAUL MINAS KUYUMJIAN", LARRY HEAMAN
}

\begin{abstract}
Mafic dikes are abundant in the Quadrilátero Ferrífero and Southern Espinhaço regions, southeastern State of Minas Gerais. The dikes range from 1,7 Ga to $120 \mathrm{Ma}$ and form at least three swarms that differ in trend, mineralogy, composition and age. The oldest swarnrhas a baddeleyite $\mathrm{U} / \mathrm{Pb}$ age of 1,7 Ga (Ibirité Gabbro) and $\mathrm{K}$ - Ar age of about $1,7-1,5 \mathrm{Ga}$ (schists), trends north-south, the dikes vary in width from centimeters to meters, and are deformed and metamorphosed to cnlorite-sericke-quartz-plagioclase schist or are some meters wide and contain plagioclase, augite,- ilmenite, quartz and traces of baddeleyite. These dikes are enriched in LREE $[(\mathrm{La} / \mathrm{Sm}) \mathrm{N}=3,68-6.39]$, and depleted to enriched in HREE [(Dy/Yb)N $=0,82-1,29]$. These dikes (and sills also) are related to the opening of the Espinhaço Basin.

Nprthwest-and northeast-trending mafic dikes have a U/Pb age of about $906 \mathrm{Ma}$. They are commonly ten meters wide, and are of metagabbro with relict igneous textures and containing altered plagioclase (Ans-52), amphibole, epidote, ilmenite, quartz, and apatite. Samples of this dike swarm are slightly enriched in LREE and HREE with a $(\mathrm{La} / \mathrm{Sm}) \mathrm{N}=1.32$ to 2.2 and $(\mathrm{Dy} / \mathrm{Yb}) \mathrm{N}=1.36$ to 1.88 , respectively, and also show small negative europium anomaly. Sills, stocks, plugs, laccoliths and phaccoliths with compositions similar to dikes are present in the study area. The Sm-Nd data indicate negative ENU (T) $\left(+1.34\right.$ and - 3.20) and model ages $\left(\mathrm{T}_{\mathrm{DM}}\right)$ of ca. 2.59 and $1.35 \mathrm{Ga}$. These dikes may be related the first stages of the Panafrican/Brasiliano event.

North-south, east-west, northwest and northeast trending Phanerozoic dikes are of diabase composed of plagioclase $($ An«)), augite and magnetite. They show moderate LREE $(\mathrm{La} / \mathrm{Sm}) \mathrm{N}=2,53$, and slight HREE enrichments, (Dy/Yb)N $=1,47$. These dikes have a $\mathrm{K} / \mathrm{Ar}$ age of about $120 \mathrm{Ma}$ and may be related to the fragmentation of Gondwanaland The Quadrilátero Ferrífero and Southern Espinhaço dikes are tholeitic and exhibit prominent iron-enrichment. Some trace element distributions are similar to continental rift basalts. They represent significant amounts of mafic melts formed during the beginning of ample extensional tectonic regimes. There are significant variations in incompatible and partially compatible element abundances both within and between dike swarms, which is due not only to heterogeneous mantle sources, but also to crustal contamination.
\end{abstract}

RESUMO ENXAMES DE DIQUES MÁFICOS DO QUADRILÁTERO FERRÍFERO E ESPINHAÇO MERIDIONAL, MINAS GERAIS, BRASIL Enxames de diques máficos são abundantes no Quadrilátero Ferrífero Quadrilátero Ferrífero e Espinhaço Meridional, sudeste de Minas Gerais. Três eventos de magmatismo básico ocorrem nestas regiões, cada qual com feições estruturais, petrográficas, geoquímicas e geocronológicas próprias. $\mathrm{O}$ enxame mais antigo tem idade de $1,7-1,5 \mathrm{Ga}$ ( $\mathrm{U} / \mathrm{P}_{\text {badeléta }}$-Ibirité Gabbro e $\mathrm{K}-\mathrm{Ar}_{\mathrm{RT}}$-xistos) e ocorre como sills e diques de direção NS, deformados e metamorfisados, ou como diques não deformados com a textura ignea preservada. Os deformados são clorita-sericita-quartzo-plagioclásio xistos, enquanto os indeformados são gabros com plagioclásio (Anse), augita, ilmenita, quartzo e tracos de badeleita. Esses diques mostram um enriquecimento em ETRL $[(\mathrm{La} / \mathrm{Sm}) \mathrm{N}=3,68-6,39]$ e um empobrecimento ou leve enriquecimento em ETRP $[(\mathrm{Dy} / \mathrm{Yb}) \mathrm{N}=0,82-1,29]$. Estes corpos são relacionados com a abertura da bacia Espinhaço. Diques NW e NE e idade U/Pb em torno de $906 \mathrm{Ma}$, possuem largura variável, estão deformados e possuem textura ignea parcialmente preservada, exceto em zonas de baixa deformação, onde apenas as bordas estão foliadas. São metagabros com plagioclásio (Ans2,5), augita, ilmenita e traços de apatita e quartzo, muitas vezes transformados em um agregado de tremolita-actinolita ou hornblenda e zoisita/clinozoisita. Amostras destes diques mostram um enriquecimento em ETRL e ETRP $((\mathrm{La} / \mathrm{Sm}) \mathrm{N}=1,34$ a 2,2 e $(\mathrm{Dy} / \mathrm{Yb}) \mathrm{N})=1,36$ a 138). Os dados $\mathrm{Sm} / \mathrm{Nd}$ apontam valores de $\mathrm{ENCJ}=+1,34 \mathrm{e}-3,20 \mathrm{e}$ idades modelos $\left(\mathrm{T}_{\mathrm{DM}}\right)=2,59 \mathrm{e} 1,35 \mathrm{Ga}$. Estes corpos são relacionados aos primeiros estágios do evento Panafricano/Brasiliano.

Os diques mais jovens possuem idade aproximada de $120 \mathrm{Ma}$, possuem direções variáveis, estão indeformados e consistem de diabásios com plagioclásio (Anóo.os), augita e magnetita. Esters diques mostram enriquecimento em ETRL e pequeno em ETRP $\left.\left((\mathrm{La} / \mathrm{Sm}) \mathrm{N}=2,53 \mathrm{e}(\mathrm{Dy} / \mathrm{Yb})_{\mathrm{N}}=1,47\right)\right)$ e se relacionam à fragmentação do Supercontinente Gondwana.

Os diques máficos de ambas regiões possuem caráter toleitico, com proeminente enriquecimento em ferro, representam volumosas fusões mantélicas que marcam o inicio de amplos regimes distensivos. As suas características químicas sugerem uma afinidade com basaltos intraplaca continental. Variações significativas no comportamento de elementos traço incompatíveis e parcialmente compatíveis dentro e entre os enxames são interpretadas como uma combinação de processos magmáticos, evolução a partir de fonte mantélica heterogênea e contaminação crustal.

INTRODUCTION Mafic dike swarms occur in all continents and have been generated since the Archean (Halls 1982). They represent significant amounts of mafic melts that cross the crust marking the beginning of ample extension tectonic regimes.

The mineralogy and chemical composition of Precambrian dikes are very similar to Phanerozoic flood basalt (e.g Deccan, Karoo, Columbia River), and can represent the source of volcanic centers removed by erosion during periods of continental emergence (Halls 1982, Tarney \& Weaver 1987, FrizTopfer 1991).
A variety of geological methods have been applied in the study of dikes, mainly aiming their petrogenetic evolution. Dike swarms, mainly those of Precambrian age, are essential tools for the understanding of the geodynamic process, and certainly, are sensitive indicators of the geological processes that took place during the history of the earth.

This paper describes the mafic dike swarms of the Quadrilátero Ferrífero and Southern Espinhaço situated in the southern portion of the São Francisco Craton (Fig. 1). Our main aim is to characterizate the basic pulses that occur in

* Instituto de Geociências, Universidade de Brasília, Campus Asa Norte, Brasília DF, 70919-970

** Instituto de geociências, Universidade Federal do Rio Grande do Sul, Campus do Vale, Av. Bento Gonçalves 9500, Porto Alegre, RS

*** Royal Ontario Museum, Ontario, Canada 
these regions, by the investigation of their field relationships, petrography and geochemistry.

GEOLOGICAL SETTING The Quadrilátero Ferrífero and Southern Espinhaço are key areas to understand the tectonic evolution of the São Francisco Craton.

Quadrilatero Ferrífero The Quadrilatero Ferrífero and adjacent regions contain Archean to Paleoproterozoic granite-gneissic terranes and greenstone belts (Rio das Velhas Supergroup), and supracrustal rocks of Paleoproterozoic (Minas Supergroup), Paleoproterozoic-Mesoproterozoic (Espinhaço Supergroup) and Neoproterozoic (São Francisco Supergroup) ages (Fig. 1 and 2).

The supracrustal units of the Minas and .Rio das Velhas Supergroups surround and are surrounded by granite-gneiss domes. These domes are of polydeformed gneiss, metatonalite to metagranite, amphibole, metaultramafic rocks, as well as pegmatites, metamorphosed under amphibolite facies during Archean and Tranzamazonic times (Cordani et al. 1980, Machado et al. 1989a, Chemale et al. 1994).

The Rio das Velhas Supergroup is divided into the Nova Lima Group, consisting of a lower ultramafic unit, an intermediate felsic-mafic unit, and an upper chemical unit (Ladeira 1980, Schorscher 1978), and the Maquine Group, which consists of quartzites (Dorr 1969) (Fig. 2).

The overlying Paleoproterozoic Minas Supergroup consists of a lower clastic unit (Caraça Group), a middle clasticchemical unit (BIF-bearing Itabira Group), and an upper chemical-clastic unit (Piracicaba Group).

The Paleoproterozoic-Mesoproterozoic Espinhaço Supergroup is exposed in the northern portion of the Quadrilátero Ferrífero, in the Cambotas mountains (Fig. 2) (Schorscher et al.; 1982;Marshak\&Alkmim 1989, Chemale Jr. etal. 1994), and consists of metaconglomerates and quartzites.

Both the Archean and Paleoproterozoic units display a complex metamorphic and deformational history (Dorr 1969, Marshak \& Alkmim 1989, Chemale et al. 1994). Several bodies of basic dikes occur in the Quadrilátero Ferrífero, and are intimately related with the different tectonic-metamorphic events of the region. The main basic dikes are shown in figure 2 .

Southern Espinhaço The Southern Espinhaço is a part of the N-S elongated Aracuai belt (Uhlein et al. 1986, Chemale Jr. etal. 1993) (Figs. 1 and 3), and is represented by alluvial, coastal eolian, deltaic, and marine sediments of the Espinhaço Supergroup. Volcanic rocks (e.g. Conceição do Mato Dentro rhyolite) of 1,75 Ga (Brito Neves et al. 1979) occur as intercalation at the base of the Supergroup, and mafic rocks intruded the unit before the Brasiliano Cycle (Silva et al. 199la and b).

Exposures of Archaean granite-gneiss complexes and volcano-sedimentary rocks occur within this belt. The former consist of polydeformed gneisses, with minor migmatites and amphibolite. The later contains greenschists, talc-chlorite schist, sericite schist, banded iron formations, sericite quartzite, quartz schist, and quartzite.

The Southern Espinhaço region is rimmed by metasedimentary rocks of the Salinas Group, of Brasiliano age to the north, Archean to Paleoproterozoic granite-gneiss complexes and small remnants of rocks belonging to the Minas and Rio das Velhas Supergroups, strongly reworked during the Brasiliano Cycle, to the East, and deformed Neoproterozoic sedimentary covers of the Sao Francisco Supergroup (Macaúbas and Bambui Groups) to the West. The later overlies the Espinhaço Supergroup by angular and erosive unconformities (Pflug \& Renger 1973).

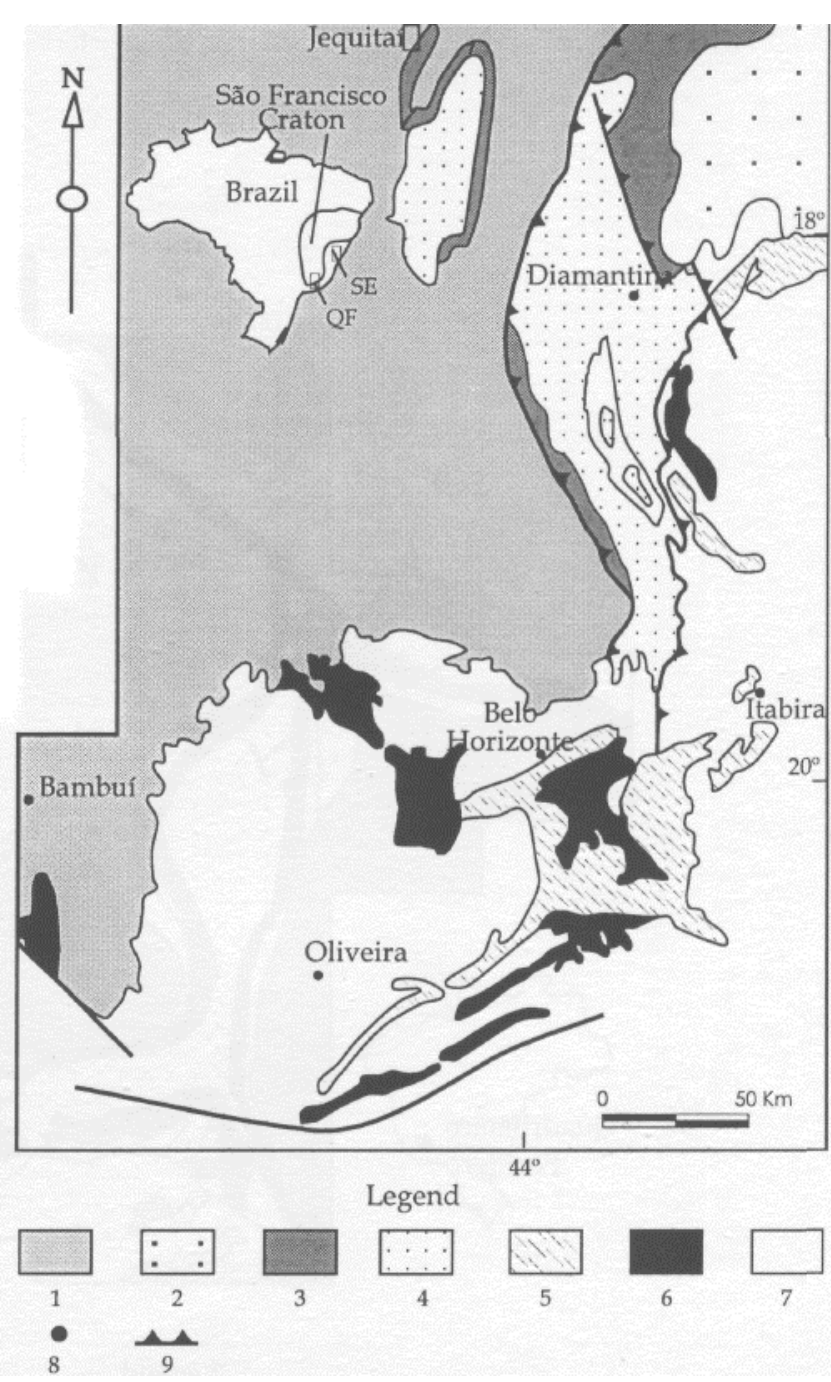

Figure 1 - Geological map of southern portions of the São Francisco Craton and southeastern of the Aracuai Belt, after Chemale et al. (1991). (1) Bambui Group; (2) Salinas Group; (3) Macaúbas Group; (4) Espinhaço Supergroup; (5) Minas Supergroup; (6) Archaean Greenstone Belts; (7) Granitegneis terrains; (8) Towns; (9) Thrust faults

DIKE SWARM GEOLOGY

Types of Intrusions

Considering their stratigraphic positions, compositions and ages, we divide the mafic swarm of the Quadrilátero Ferrífero and Southern Espinhaço into three types:

(1) N-S oriented, centimetric to metric wide dikes, sills, and stocks, either deformed, metamorphosed and lacking igneous features, or undeformed and with preserved igneous texture. In the Southern Espinhaço, these dikes are restricted to the sin-rift sequence of the Espinhaço basin; (2) Dikes, sills, stocks, laccoliths, lopoliths or plugs, with northeast, northsouth, northwest, locally east-west directions. The width of the dikes and sills is decimetric to metric, and the hypoabyssal bodies vary from metric up to $5 \mathrm{~km}$ in diameter. Intrusions of this type cut through all rocks of the Minas and Espinhaço Supergroups;

(3) Decimeter to centimeter wide, north-south, east-west, northwest, and northeast oriented, undeformed and non metamorphosed dikes with fully preserved igneous texture. These dikes intruded into all Precambrian rocks of the region.

Contacts The contact relationships of the intrusions with the country rocks are not always clear, due to alteration and 


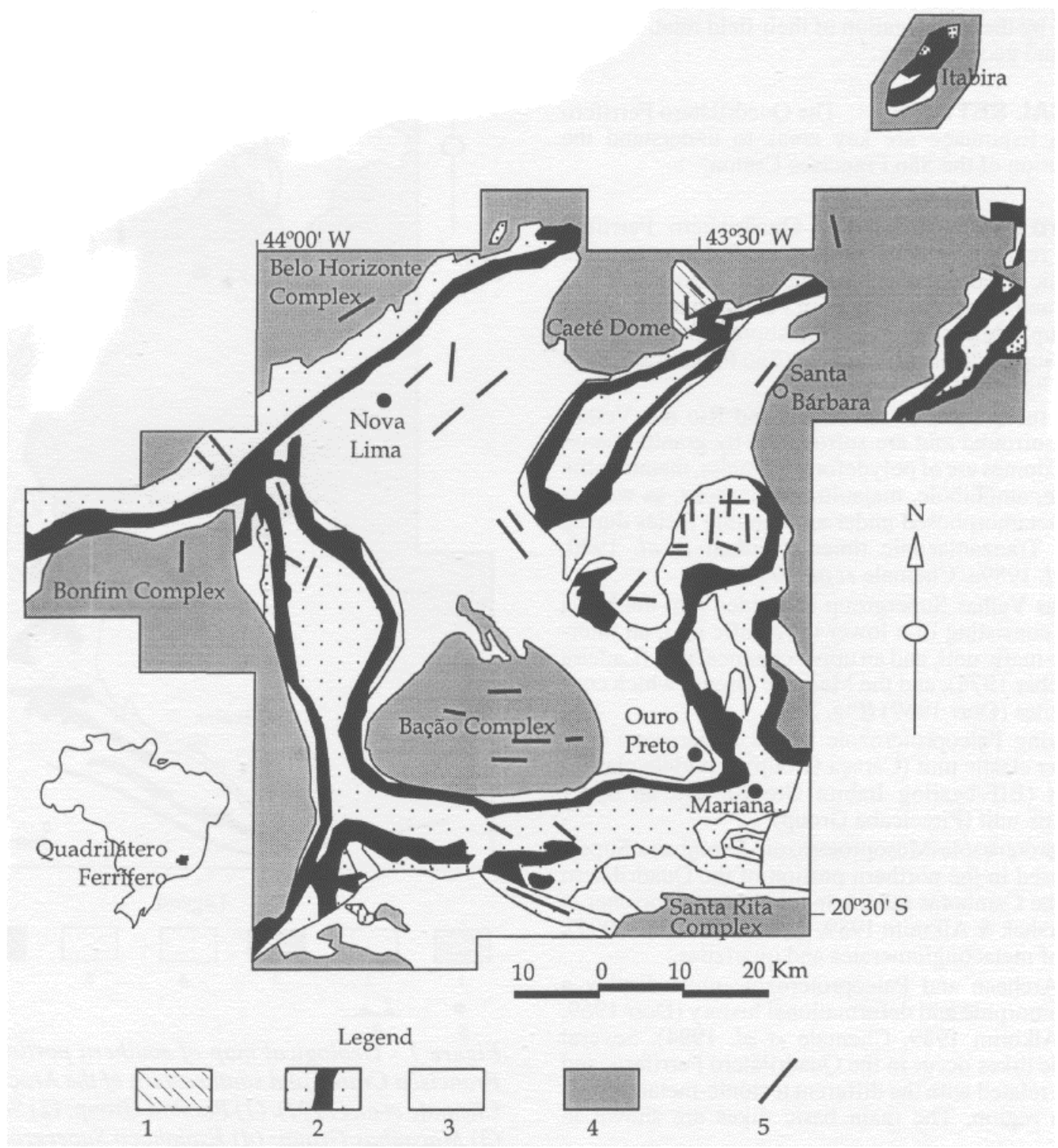

Figure 2 - Geologic map of the Quadrilátero Ferrifero. (1) Espinhaço Supergroup, (2) Minas Supergroup, (3) Archaean Greenstone Belts, (4) Granite-gnaiss terrains, (5) Mafic Dikes (modified after Dorr J969)

scarcity of outcrops. When preserved, the contacts are discordant in low strain zones or tectonic in high strain zones. In the Southern Espinhaço, the contacts of the larger mafic bodies are generally covered by sediments.

Tectonic contacts have a North-South orientation, similar to those of the regional structures. Recrystallization of both the intrusion and the host rocks at the contacts is generally conspicuous, as indicated by the silicification of the former, and the chloritization of quartzites. Transtension zones are commonly invaded by quartz veinlets.

Structural Features The structural features observed in the mafic intrusions include anastomosed mylonitic foliation, centimetric tension gashes, fractures (conjugated or not), thrust, normal and transcurrent faults, and mineral, stretching and crenulation lineations.

The dikes have a border foliation parallel to the regional foliation of the Minas and Espinhaço Supergroups. The mylonitic foliation in the hosts varies between $\mathrm{N} 22^{\circ} \mathrm{E} / 65^{\circ} \mathrm{SE}$ and $\mathrm{N} 74^{\circ} \mathrm{E} / 44^{\circ} \mathrm{SE}$, while at the border of the mafic bodies it varies between $\mathrm{N} 40^{\circ} \mathrm{E} / 22^{\circ} \mathrm{SE}$ and $\mathrm{N} 55^{\circ} \mathrm{E} / 30^{\circ} \mathrm{SE}$. The mineral and stretching lineations both in the dikes and host rocks are approximately east-west, while the crenulation lineation is north-south.

The inverse faults are N-S and define the contacts of the basic bodies with the hosts. In these cases, the borders of the bodies are either sheared or, under shallower crustal levels, brecciated. In low strain domains, the basic bodies are only incipiently affected by regional deformation, while the host rocks still preserve their primary features.

In the western border of the Southern Espinhaço, the mafic intrusions are related to transcurrent and normal faults. In the southern portions of the eastern border, the contacts are apparently tectonic and marked by inverse and thrust faults.

The kinematic indicators associated to tension gashes indicate that the main tectonic transport took place from east to west, as also previously deduced by Ulhein etal. (1986), and Chemaleeftf/. (1993).

Petrography GROUP I Rocks of this group are quartz-chlorite schist and chlorite-chloritoid-sericite schist, as 
well as locally undeformed, melanocratic, equigranular, coarse to medium-grained gabbro and microgabbro.

The quartz-chlorite schists are light green to greyish, fine grained, and contain chlorite (50-55\%), quartz (30-35\%) with minor ilmenite (10\%) and plagioclase (Ano5). Relicts of primary feldspar may occur. Accessories include apatite and zircon. Leucoxene is the most abundant secondary mineral and formed after ilmenite. Besides deformation and metamorphism, samples of this group also underwent metasomatism, as indicated by the presence of sericite.

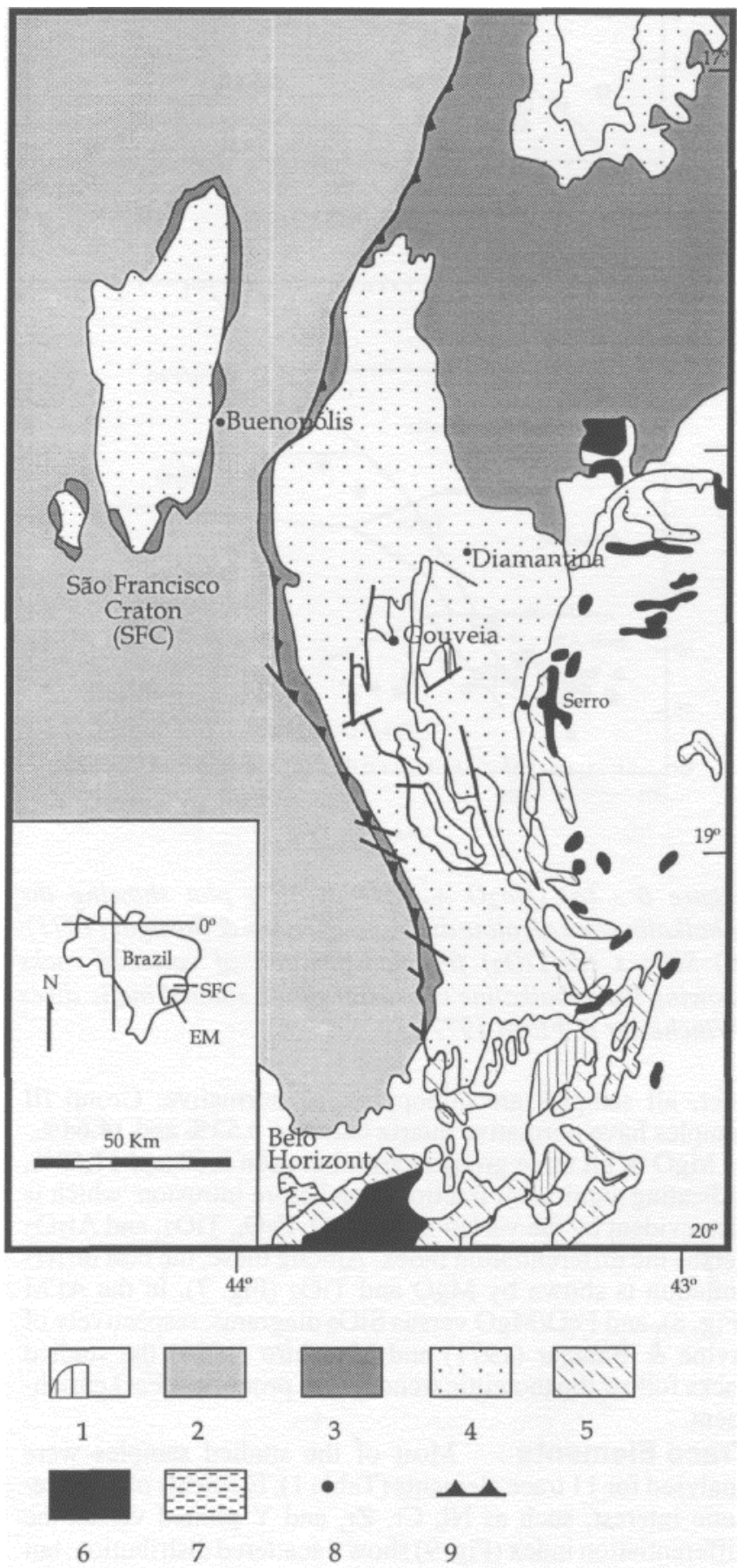

Figure 3 - Geologic map of Southern Espinhaço, (l) metabasics; (2) Mantiqueira Province with Brasiliano granites; (3) Bambui Group; (4) Macaúbas Group; (5) Espinhaço Super group; (6) Minas Supergroup; (7) Granite-gnaiss terrains; (8) Towns; (9) Thrusts (Modified from Dossin 1983)
The chlorite-chloritoid-sericite schists are greenish. Primary features such as millimetric to centimetric vesicles occur locally. These schists have granoblastic and lepidoblastic textures, are fine grained, and are formed by sericite $(65 \%)$, chlorite $(15 \%)$, magnetite $(5 \%)$, tourmaline $(2-5 \%)$, titanite $(1 \%)$, and zircon $(1 \%)$. When present, the proportions of chloritoid may be as high as $10 \%$.

The gabbros and microgabbros have intergranular, locally also ophitic textures. They consist of labradorite (An56-60) laths, subhedral to anhedral augite, and up to $5 \%$ of subhedral to anhedral ilmenite. The plagioclase and augite crystals are slightly deformed. Trace amounts of potassium feldspar may occur and is myrmekitic at the contact with labradorite. Apatite, quartz, and locally also zircon and baddeleyite are accessories. Quartz, in particular, occurs either as anhedral isolated and interstitial crystals, or associated to potassium feldspar as myrmekitic and micrographic intergrowths.

Chemical data of pyroxene shows that its composition lies between intermediate pigeonite and subcalcic augite (Fig. 4). Plotted in the $\mathrm{CaMgSiO}_{3}-\mathrm{CaFeSiO}_{3}-\mathrm{MgSiOs}-\mathrm{FeSiO}_{3}$ system (Fig. 5), at several temperatures and $1 \mathrm{~atm}$ pressure (Linsdey 1983), the analytical data indicate that the pyroxene crystallized between $1100^{\circ}$ and $1200^{\circ} \mathrm{C}$, with an approximate average of $1150^{\circ} \mathrm{C}$.

GROUP II Rocks belonging to Group II are the most abundant in the Quadrilátero Ferrífero and Southern Espinhaço. They consist of massive, melanocratic, medium grained metagabbros, locally with preserved ophitic and sub-ophitic primary textures. Thin bodies are commonly foliated.

The metagabbros consist of $50-55 \%$ of plagioclase laths (An60.05 to Anos), with interstitial subhedral to anhedral augite $(35-40 \%)$ and subhedral to anhedral ilmenite (3-5\%). The pyroxene is commonly uratilitized into actinolite-tremolite or hornblende, and plagioclase saussuritized to zoisite/ clinozoisite. Quartz, apatite, potassium feldspar, biotite, and zircon are accessories.

GROUP III Rocks of this group are melanocratic, medium to fine grained, massive diabase dikes with intergranular texture characterized by lath-shaped labradorite (An60,50), with interstitial augite. The texture is locally ophitic. Magnetite, pyrite, ilmenite, olivine, apatite, titanite, and quartz are accessories. Leucoxene occurs as alteration of ilmenite.

Plagioclase (45-65\%) is the most abundant mineral in these rocks. The mineral occurs as subhedral, clean, slightly sericitized, zoned and twinned crystals, commonly with fractures, undulate extinction and corrosion bays.

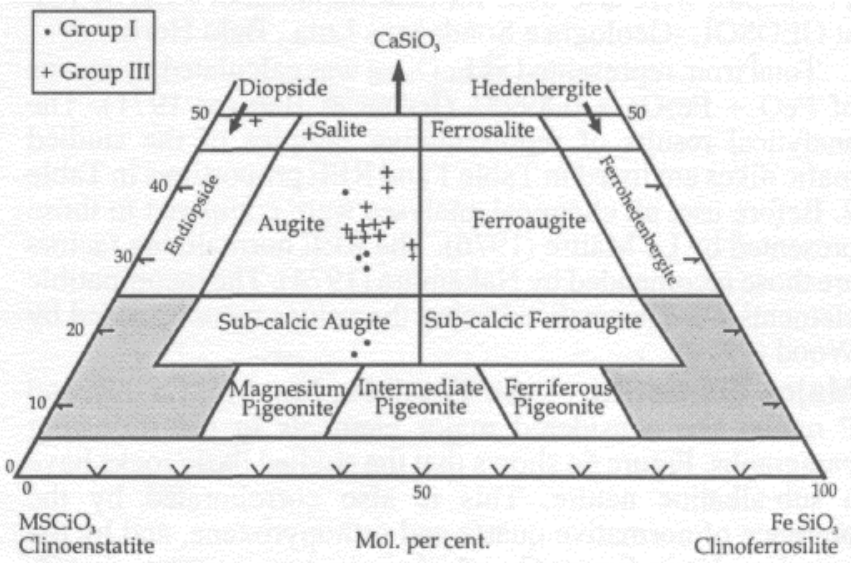

Figure 4 - Classification of pyroxenes of mafic dike swarms plotted in the system $\mathrm{CaMgSiO}_{3}-\mathrm{CaFeSiO}_{3}-\mathrm{MgSiO} 3$ $\mathrm{FeSiO}_{3}$ ( Poldervaart and Hess 1951). 


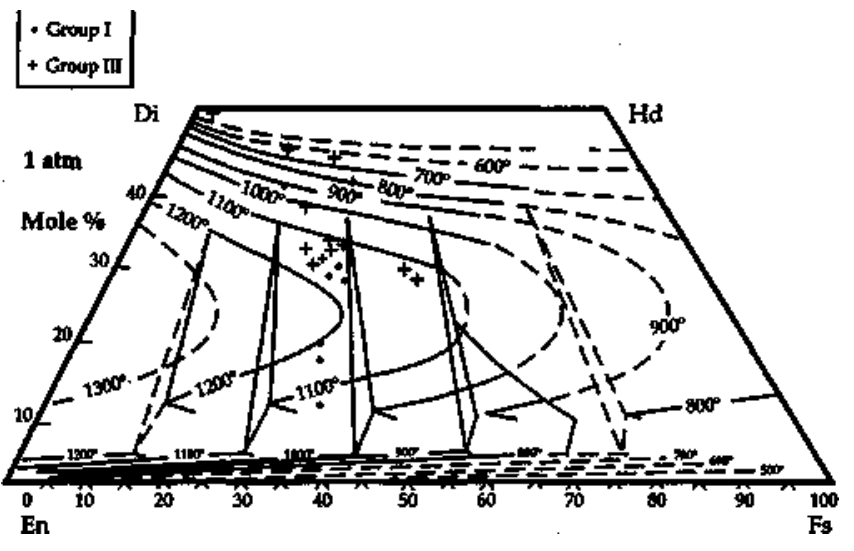

Figure 5 -Pyroxene compositions of the studied mafic dikes plotted in the system Di-Hd-Fs-En at latm (Linsdley 1983).

The clinopyroxene $(15-45 \%)$ is subhedral to anhedral, locally euhedral, and presents lamellar twinning and undulating extintion. Chemical data of the pyroxene are compatible with the composition of augite (Fig. 4), and, as in figure 5, the mineral crystallized at approximately $1150^{\circ} \mathrm{C}$.

Magnetite (6-10\%) occurs either as skeletal and sutured crystals, bordered by titanite and leucoxene, or as lath-shaped crystals. Microprobe analysis of magnetite shows that it may contain up to $2.6 \%$ of vanadium. Pyrite (3-5\%) occurs as cubic disseminated crystals. Olivines is generally altered to iddingsite, but relicts are rich in fayalite (Fa49-5g). Quartz is rare and occurs as interstitial anhedral crystals. Apatite is the main accessory and is-euhedral to needdle shaped.

Rocks of this group may locally be hyalocrystalline, with a matrix composed of plagioclase, lath-shaped and skeletal magnetite, and glass with the composition of subcalcic ferrous-augite. When hypocrystalline, they contain submillimetric vesicles filled with partially altered volcanic glass.

PETROCHEMISTRY About 20 mafic bodies were sampled for chemical analysis, totaling 76 samples. After cleaning, grinding and reduced to powder, the samples were analyzed for major, minor and trace element determinations in the Geochemical Laboratory of the University of Brasilia. $\mathrm{FeO}$ was determined by volumetry, $\mathrm{K}_{2} \mathrm{O}$ and $\mathrm{Na}_{2} \mathrm{O}$ by flame photometry, LOI by gravimetry, and the remaining major and minor element oxides, as well as selected trace elements by inductivelly coupled plasma emission (ICP). Among theses, 11 samples were also used for determination of REE by ICP at GEOSOL -Geologia e Sondagens Ltda., Belo Horizonte.

Total iron, represented as $\mathrm{FeO}_{\text {total }}$ was calculated by means of $\mathrm{FeO}+\mathrm{Fe}_{2} \mathrm{O}_{3} \times 0.89981$ (Irvine \& Baragar 1971). The analytical results of representative samples of the studied mafic dikes are listed in Table 1 and REE proportions in Table 2 . Before use, all chemical analyses were compared to those presented by Le Maitre (1976). The REE normalizing factors are those recomended by Nakamura (1974). The incompatible elements were normalized using the values recommended by Wood (1979).

Major Elements $\mathrm{Si}, \mathrm{Ti}, \mathrm{Al}, \mathrm{Fe}, \mathrm{Mn}, \mathrm{Mg}, \mathrm{Ca}, \mathrm{Na}$, and $\mathrm{P}$ oxides are considered major elements in the following paragraphs. Figure 6a shows that the studied basic rocks have a sub-alkaline nature. This is also corroborated by the presence of normative quartz and orthopyroxene, and by the samples plot in figure $6 \mathrm{~b}$, which in its turn suggests that $\mathrm{Zr}$ and Ti had low mobility since intrusion.

Normative quartz of Group I samples is between $2.32 \%$ and $14.05 \%$, while in Group II it averages $6.45 \%$. When not quartz normative, they have 0.09 to $14.1 \%$ normative olivine. How-
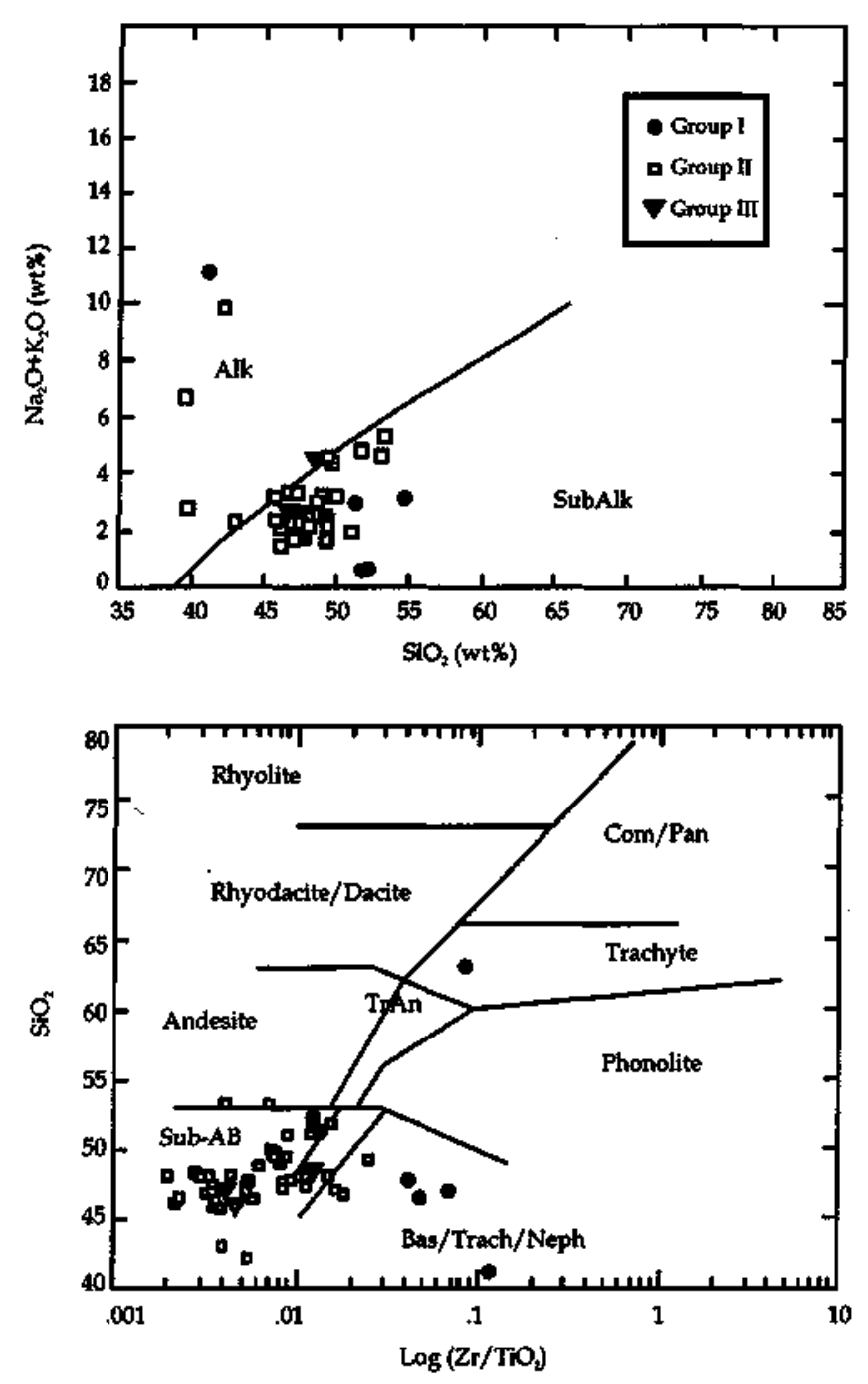

Figure 6 - (a) $\left(\mathrm{Na}_{2} \mathrm{O}+\mathrm{K}_{2} \mathrm{O}\right) \times \mathrm{SiO} 2$ plot showing the subalkaline andalcaline divisions of Irvine \& Baragar (1971). (b) $\mathrm{SiO}_{2} \times\left(\mathrm{Zr} / \mathrm{TiO}_{2}\right)$ for classification of volcanic rocks showing the subalkaline character of the studied mafic dikes (Winchester \& Floyd 1977).

ever, all samples are clinopyroxene normative. Group III samples have normative quartz between $5.53 \%$ and $14.64 \%$.

$\mathrm{MgO}$ of all three groups varies between 2.68 and $11.68 \%$, indicating significant fractionation before intrusion, which is also evident by the variation of $\mathrm{MgO}, \mathrm{FeO}_{\mathrm{t}}, \mathrm{TiO}_{2}$, and $\mathrm{Al}_{2} \mathrm{O}_{3}$ versus the differentiation index. Among these, the best differentiation is shown by $\mathrm{MgO}$ and TÍÜ2 (Fig. 7). In the AFM (Fig. 8), and $\mathrm{FeO} / \mathrm{MgO}$ versus $\mathrm{SIO}_{2}$ diagrams, respectively of Irvine \& Baragar (1971) and Myashiro (1974) the studied rocks follow the tholeiitic trend with a prominent $\mathrm{FeO}$ enrichment.

Trace Elements Most of the studied samples were analysed for 11 trace elements (Table 1). Elements of petrogenetic interest, such as $\mathrm{Ni}, \mathrm{Cr}, \mathrm{Zr}$, and $\mathrm{Y}$ plotted versus the differentiation index (Fig. 9) show a scattered distribution, but with decreasing contents of $\mathrm{Cr}$ and $\mathrm{Ni}$, and increasing $\mathrm{Y}$ and $\mathrm{Zr}$.

Due to the low mobility of $\mathrm{Ti}$ and $\mathrm{Zr}$ during alteration and low grade metamorphism, as well as the small variation of their ratios during mantle partial melting and formation of basaltic liquids, their ratios closely reflect the composition of the sources (Condie 1985a). In both studied regions, Group I 
Table l- Chemical composition of mafic dikes of the Quadrilátero Ferrifero e Southern Espinhaço regions. *GroupI, Group II, **GroupIII

\begin{tabular}{|c|c|c|c|c|c|c|c|c|c|c|c|c|c|c|c|}
\hline & ands & macta & +lolos & tul & 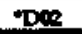 & $\operatorname{Dos}$ & Dirkol & Drap & Fin & Frid & DATM & DATO3 & covor & Pag & sing \\
\hline $60_{2}$ & 57,88 & 529 & 46,11 & 4,28 & 458 & 4,05 & 472 & 46,4 & 46,91 & $\infty, \pi$ & 4,26 & 48,12 & 39,73 & 600 & 43 \\
\hline$T O_{2}$ & 1,57 & 136 & 400 & 1,28 & 2,65 & 1,56 & 2,11 & 1,09 & 104 & 2,11 & 2,55 & 1,24 & 1,18 & 1,95 & 1,19 \\
\hline $\mathrm{ALO}$ & 13,1 & 13,17 & 1212 & 2051 & 12,02 & 1262 & 13,4 & 15,1 & 16,09 & 13.4 & 1399 & 13,69 & 20,00 & 12,74 & 256 \\
\hline FeO & 1401 & $14 t$ & 125 & $3, a$ & 1,71 & 1,6 & $10,0 \mathrm{~s}$ & 40 & 0,26 & 3,08 & 9,17 & 7,8 & 10,84 & 21,99 & 1006 \\
\hline Mno & 0,22 & 0,21 & 0,24 & 0,1 & 0,19 & 0,1 & 0.2 & 0,19 & 0,2 & 0,19 & 0,17 & 017 & 02 & 0,17 & 0,21 \\
\hline $\mathrm{MgO}$ & 8,02 & 7,91 & 476 & 5,25 & 6,74 & 6,08 & 7,16 & $s, a$ & 9,1 & 7,14 & 7,35 & 9,13 & $7 \omega$ & 673 & 40 \\
\hline $\mathrm{CwO}$ & 0,22 & 0,28 & 97 & 01 & 964 & 937 & $11, \$$ & 11,6 & 12,02 & 10,74 & 10,43 & 11,4 & 1,05 & 935 & 2,22 \\
\hline Nero & 0.29 & 0,37 & 211 & 06 & 250 & 236 & 1.94 & 1 & $1, s$ & 2,13 & 2,13 & 137 & $0 \beta \theta$ & 1,81 & 0,9 \\
\hline 180 & 0,19 & 0,24 & 0,79 & 10,52 & 0 & 0,5 & 0,006 & 0,6 & 0,80 & 0,68 & 0/4 & 029 & 397 & 05 & 975 \\
\hline $\mathrm{P}_{2} \mathrm{O}_{3}$ & 0,14 & 0,15 & 09 & 0,9 & 15 & $1 / 4$ & 0,24 & 022 & 0,14 & 0,14 & 0,14 & 0,16 & 0,3 & 0,24 & 0,22 \\
\hline $\mathbf{P P}$ & 7,1 & 7,07 & 264 & $\$ \beta$ & 5,16 & $\$ 20$ & 3,11 & 406 & $2, \not \beta 7$ & 206 & 42 & 3,52 & $2 \theta$ & 9,67 & 4,05 \\
\hline Tase-1 & $1040 \%$ & 10,57 & 9,9 & ienst & 100,12 & 10,1 & 101,47 & 100,5 & 100,37 & 100,7 & 1095 & 160,37 & 925 & 148 & 1047 \\
\hline $\mathbf{Z n}$ & 220 & 218 & 210 & $\pi$ & 150 & 76 & 109 & 120 & 101 & 95 & 90 & 85 & 120 & 500 & 135 \\
\hline co & 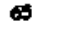 & 61 & 200 & $\$ 8$ & 32 & 31 & $\Delta t$ & 70 & $\pi$ & 77 & $\mathbf{8 0}$ & 100 & $\theta$ & 4 & $\pi$ \\
\hline $\mathrm{Cr}$ & 52 & $\varphi$ & 14 & 35 & 30 & 27 & 100 & 94 & 200 & 200 & 180 & 165 & 14 & $1 \%$ & 9 \\
\hline $\boldsymbol{v}$ & 3 & 30 & 793 & & & & & & & & & & & & \\
\hline B. & p & 5 & 2 & 5 & 5 & 6 & 5 & 5 & 5 & 5 & 5 & 5 & 5 & 5 & 5 \\
\hline $\mathrm{Cu}$ & 6 & 100 & 12 & 19 & 111 & 9 & 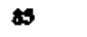 & 92 . & 74 & 69 & 191 & 60 & 100 & 90 & $m$ \\
\hline $\mathbf{z}_{\boldsymbol{r}}$ & 120 & 116 & 113 & 970 & $+\infty$ & 800 & 48 & 38 & $x$ & 23 & 27 & 111 & 36 & $\theta$ & 4 \\
\hline $\mathbf{Y}$ & 20 & $\boldsymbol{z}$ & 40 & $\$ 2$ & $\theta$ & 46 & 25 & $\mathbf{2}$ & 12 & 12 & 15 & 16 & 25 & 46 & 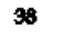 \\
\hline s. & 5 & $s$ & 199 & $\omega$ & 76 & 1 & $2 \mathrm{t1}$ & 235 & 364 & 366 & 39 & 36 & 20 & 2 & 20 \\
\hline En & 0 & 100 & 20 & 1651 & 3000 & 1052 & 19 & 169 & 18 & 115 & $\mathbf{s}$ & 89 & 100 & 17 & 21 \\
\hline$F \sigma$ & 16,95 & 16,86 & 16.64 & 2,90 & 14 & 13,12 & 1400 & 10,02 & 10,39 & 13,0 & 12,97 & 10 & 13,9 & 142 & 1024 \\
\hline $\mathbf{F} / \mathbf{F}+\mathbf{M}$ & D, & Q6s. & 078 & $0 \times 3$ & 0.6 & 0,68 & 067 & 05 & ose & oss & 0,64 & 085 & 0 & 0,8 & $\mathbf{0 . 8}$ \\
\hline
\end{tabular}

\begin{tabular}{|c|c|c|c|c|c|c|c|c|c|c|c|c|c|c|c|}
\hline & $\sin$ & $\sec$ & entor & $\sin 1$ & gon & carne & CAPH & CAMP & Catu & Hes & $\operatorname{sen}$ & $\cos$ & cos: & cots & cos: \\
\hline $\mathrm{SO}_{2}$ & 46,2 & 46/46 & 46,23 & 90,2 & 48,01 & 0979 & 45:84 & 46,62 & 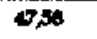 & 46,92 & 0,96 & 8,13 & 0,12 & $\theta, 56$ & $\theta / 8$ \\
\hline $\mathbf{T O}$ & 1,90 & 1,91 & 2,65 & 392 & 1,66 & 1,74 & 3,92 & 2,00 & 2,06 & 1,25 & 1,67 & 1,6 & 1,57 & 1,58 & 1,88 \\
\hline Albo & 1266 & 1118 & $120 \%$ & 125 & 136 & 148 & 12,76 & 13,56 & 3309 & 1976 & 145 & 1406 & 13,9 & 19,7 & 13,59 \\
\hline FeO & 1259 & 11,3 & 11,00 & B.66 & 8,16 & 11,34 & 11,6 & 9,4 & 9,8 & 8,67 & $7 \infty 6$ & 787 & 7,78 & 0,1 & 0,1 \\
\hline MnO & 0,18 & 0,21 & 0,19 & 0,00 & 0,16 & 0,2 & 0,21 & $0 ; 2$ & 0,2 & 0,19 & 0,16 & 0,18 & 0,17 & 017 & 0,18 \\
\hline $\mathrm{M}_{8} \mathrm{O}$ & 6,65 & 5,0 & 5,86 & 3,65 & 9,13 & 719 & 534 & 7,10 & 7,25 & 982 & 8 & 876 & 8 & 89 & 8,2 \\
\hline $0 \omega$ & 7,97 & 1000 & 928 & $7 \mathbf{8 9}$ & $120 \%$ & 79 & 904 & 107 & 10,7 & 9.91 & $11, \theta$ & 11,99 & 12,28 & 124 & 12,90 \\
\hline Nex & 2,05 & 100 & $1 / A 1$ & 299 & 2,22 & 2,05 & 2,4 & 202 & 2,02 & 157 & 2 & 1,9 & 1,3 & 1,32 & 1,20 \\
\hline $\mathrm{kOO}$ & 0,1 & 0,1 & 0,1 & 2,00 & $0 / 80$ & 0,1 & 076 & 0,48 & 066 & 0,34 & 0,34 & 0,58 & 0,99 & 0,57 & 0,39 \\
\hline $\mathrm{PxO}_{\mathrm{p}}$ & 0,28 & 0,16 & 0,2 & 0,12 & 0,19 & 0,4 & 0,6 & 0,24 & 0,26 & 0.13 & 0.21 & $0 ; 2$ & 021 & 0,18 & 0,18 \\
\hline PF & 97 & $10 / 5$ & $B, \theta$ & 5,65 & 254 & 29 & 2,92 & 2,14 & 3,06 & 387 & $2 A$ & 2,68 & 3,29 & 3,02 & $3 A$ \\
\hline Total & $\begin{array}{l}100,4 \\
194\end{array}$ & $\begin{array}{l}217 \\
217\end{array}$ & 16058 & $107 \mu$ & 1007 & 10055 & $\infty 01$ & 9,91 & 1040\% & 90 & 921 & 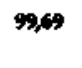 & 99,35 & $9 / 33$ & 100,25 \\
\hline $\mathbf{Z n}_{\mathbf{n}}$ & 88 & 58 & 3 & 17 & 87 & 14 & 14 & 111 & 110 & 94 & 108 & 110 & 9 & 108 & 117 \\
\hline Co & 69 & 61 & 76 & 34 & 79 & tos & 101 & $\infty$ & 82 & 94 & 73 & 7 & 12 & 77 & 80 \\
\hline cr & & & 119 & 272 & 512 & 21 & 29 & 77 & 111 & 256 & 29 & 320 & 368 & 4 & $\$ \mathbf{O I}$ \\
\hline$v$ & 1 & $\mathbf{I}$ & & & & & & & & 256 & $m$ & 290 & 201 & 260 & 26 \\
\hline B. & $s$ & 7 & 1 & 5 & 1 & 2 & 2 & 1 & 5 & 25 & 25 & 1 & 1 & 5 & 5 \\
\hline $\mathrm{Cu}$ & 60 & $\boldsymbol{z}$ & $\mathbf{3}$ & $\not 7$ & 117 & 89 & 77 & 9 & 90 & 109 & 128 & 110 & 112 & 100 & $\mathbf{1 0 2}$ \\
\hline $\mathbf{z}$ & 57 & 33 & 96 & 169 & 20 & 86 & 9 & 25 & 99 & 89 & 159 & 108 & 50 & 216 & 40 \\
\hline $\mathbf{Y}$ & 131 & 100 & so & 16 & 2 & 4 & 4 & 77 & 36 & 15 & 24 & $\mathbf{2 9}$ & $\boldsymbol{z}$ & 21 & $\mathbf{2 3}$ \\
\hline $\mathbf{s}$ & 18 & $\mathbf{2 1}$ & 48 & 50 & 218 & $n$ & 28 & 20 & 250 & 312 & 20 & 210 & 220 & 174 & 210 \\
\hline Ba & & & 14 & 329 & 118 & 365 & 565 & 170 & 192 & 324 & 14 & 15 & 145 & 137 & 200 \\
\hline $\mathrm{F} \rightarrow \boldsymbol{O}^{*}$ & $13, A$ & 12,5 & 13,76 & 15,2 & $10 \$ 9$ & $13, \pi$ & 15,46 & 19,7 & 13,22 & 11,00 & 9,6 & 9,85 & 10,35 & 10,35 & 10,46 \\
\hline $\mathrm{F} / \mathrm{F}+\mathrm{M}$ & 0.67 & 07 & 07 & 0,81 & 084 & 0,66 & 0,5 & 0,65 & 06 & 0.8 & 0,3 & 0,8 & 05 & 0,4 & 054 \\
\hline
\end{tabular}

Legnd:"Grupol Groupl * Grupoll 
Table l- Chemical composition of mafic dikes of the Quadrilátero Ferrifero e Southern Espinhaço regions. *GroupI, Group II, **GroupIII (continuation...)

\begin{tabular}{|c|c|c|c|c|c|c|c|c|c|c|c|c|c|c|c|}
\hline & RR3 & Rakep & CAMUI & TANon & TAMA & ThMllah & Tingl & There & TIMes & TMM & THeas & T1M:as & Glun & MGIVE & Honas \\
\hline 502 & $\mathbf{5 1 , 1}$ & 303 & 0,69 & 5109 & 486 & 999 & 900 & $\mathbf{3 0 , 0 1}$ & 15,0 & 4,0 & 51,1 & 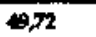 & 13 & \$56 & 4,7 \\
\hline TiOn & 2,85 & 1,62 & 1,79 & 287 & 481 & 4,81 & 3,87 & 405 & 389 & 3,2 & 3,00 & 3,4 & 21 & 1,9 & 0,07 \\
\hline Alnos & 15,43 & $19 \mathrm{~A}$ & 1290 & 149 & 1377 & 1407 & 11,7 & 11.16 & 1157 & 12,91 & 1469 & 1404 & 13,17 & 10,14 & 12,86 \\
\hline Fe200 & 3,4 & 3,39 & 262 & 3,07 & 7,25 & 11,47 & 347 & 11,6 & 2,4 & 232 & 2,37 & 2,25 & 281 & 21 & 200 \\
\hline$F \omega$ & 88 & 476 & 9,72 & $9 / 7$ & $7, \mathbf{1}$ & $5 / 4$ & 145 & 7,18 & 1492 & $11 / A 7$ & 12,06 & 12,6 & 120 & 458 & 7,59 \\
\hline $\operatorname{MnO}$ & 0,16 & 0,04 & 0,21 & 0,14 & 0,24 & 0,23 & 0,16 & 0.19 & 0,06 & 0,21 & 2,79 & 0,5 & 0,2 & 0,17 & 0,19 \\
\hline $\mathrm{MgO}$ & 285 & 1,60 & 75 & $3,6 s$ & 4 & 6,99 & $5 ; m$ & 46 & 495 & 36 & 634 & 3,00 & 6,6 & $11, \$ 6$ & 10,01 \\
\hline $\mathrm{C \omega}$ & 6,1 & 6,24 & 10,8 & 6,89 & 3,95 & 8,6 & 3,09 & 5,1 & 5,14 & 376 & 273 & 6,06 & $9, \pi$ & 12,26 & 13,04 \\
\hline $\mathbf{m a O}$ & 0,19 & 1 & 0,7 & 1,92 & 0,31 & a,A & 1,11 & $1, A$ & 1,29 & 1,79 & 0,2 & 1,7 & 0,74 & 0,0 & 0,3 \\
\hline P200 & 08 & Des & 0,19 & 107 & 0,9 & $0,5.5$ & 0,57 & 0,64 & 0,06 & $0, \theta$ & 0,28 & 1,07 & 0,22 & 0,11 & 0,1 \\
\hline $\mathbf{P F}$ & $4 \% 1$ & 3,4 & 3,14 & 2,99 & 3,6 & 4 & 3,69 & 2,0 & 83 & $1, \infty$ & 13,08 & 3,01 & 1,05 & 3,4 & 3,09 \\
\hline Totil & $\$, 19$ & 9,19 & 9,05 & 10Ak & $\$ 11$ & $\$, 13$ & 104,15 & 104 & 100,5 & $\mathbf{4}, 15$ & 10044 & 94 & 10,43 & 1097 & 10004 \\
\hline $\mathbf{Z n}$ & 2 & $z$ & 107 & 120 & 14 & 19 & 260 & 200 & 190 & 168 & 170 & 160 & 19 & $\infty$ & th \\
\hline Co & $\pi$ & 11 & 8 & 6 & 110 & 114 & 86 & 8 & 86 & $n$ & 70 & $\pi$ & 117 & 113 & 112 \\
\hline $\mathrm{Cr}$ & 9 & 35 & 123 & 30 & 50 & 32 & 30 & 20 & 30 & 20 & 20 & $\mathbf{n}$ & 157 & 72 & 300 \\
\hline $\mathbf{v}$ & 109 & 57 & $\mathbf{x}$ & 324 & $\Delta$ & 670 & 600 & 620 & 585 & 80 & $\Delta$ & 46 & 0 & $m 7$ & 200 \\
\hline Be & $\mathbf{s}$ & 5 & 3 & 1 & 1 & 1 & 3 & 3 & 3 & 3 & 3 & & 2 & 1 & 1 \\
\hline $\mathrm{Cu}$ & 9 & 15 & 79 & 10 & 52 & 52 & 360 & 30 & 345 & 158 & 179 & 188 & 54 & 42 & 4 \\
\hline $\mathbf{z}$ & 98 & 241 & 84 & 0 & 4 & 82 & 167 & 100 & 185 & 169 & 16 & 14 & 5 & 164 & 20 \\
\hline $\mathbf{Y}$ & 60 & 6 & 27 & 32 & 20 & 20 & 102 & 6 & 64 & 62 & 61 & 63 & 4 & 15 & 11 \\
\hline$\theta$ & 372 & $3 \mathbf{n}$ & 20 & $\infty 0$ & 60 & 600 & 85 & 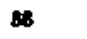 & 86 & 160 & 16 & 160 & $n$ & 209 & 906 \\
\hline Bh & $\mathbf{a 1}$ & $\operatorname{scos}$ & 200 & 500 & 175 & 116 & 20 & 200 & 260 & $3 / 5$ & 376 & 300 & 113 & 102 & $\pi$ \\
\hline Fo $\sigma^{*}$ & $11, \infty 6$ & $7, \boldsymbol{A 1}$ & 2,00 & $12+3$ & 147 & 15,76 & 17,62 & $17, a$ & 16,4 & $13, \%$ & 1416 & 14,5 & 149 & $10 / 7$ & 9,77 \\
\hline$F / F+M$ & 0,1 & 0.2 & ost & 07 & 07 & 07 & 078 & as & 077 & $0 B$ & ast & 0.03 & 0,8 & 0,62 & 0,9 \\
\hline
\end{tabular}

\begin{tabular}{|c|c|c|c|c|c|c|c|c|c|c|c|c|c|c|c|c|}
\hline & MGQB & Denerv & RmintII & Exocy & MGesto & Frn & EF⿻上丨 & Rract & exas & Futs. & MKa & $\mathrm{N}=$ & $\operatorname{Nan}$ & -FLon & \#1EAT & $-\operatorname{mon}$ \\
\hline $\mathbf{s i n}_{2}$ & 61 & 45 & $\boldsymbol{\alpha , 0 1}$ & 9,77 & 1419 & stos & 9.56 & 9,02 & atos & 030 & 469 & 44 & $\$ 06$ & 97 & 51,32 & 62.5 \\
\hline $\mathrm{THO}_{2}$ & 235 & 1,79 & 3,0 & 279 & 1,27 & 35 & 3,27 & 3,27 & 1,20 & 1,31 & 1,18 & 400 & 414 & 131 & $\mathbf{3 \mu 4}$ & 0,72 \\
\hline$A Q_{3}$ & $12 \%$ & 12,71 & 1209 & 1401 & 1922 & 1245 & 11,10 & 11,8 & 1454 & 145 & 140 & 1212 & 1224 & 1591 & 128 & 1500 \\
\hline FeO & 10,19 & 2009 & 127 & 10,49 & $5 / 46$ & 10,66 & 13,45 & 1904 & $7, \infty 8$ & 780 & 7,11 & 125 & 129 & 1,6 & 938 & 9,5 \\
\hline Mro & 021 & 0,23 & 0.25 & 0.19 & 0,18 & 0,2 & 0,23 & 0.24 & 0,17 & 0,17 & 0,14 & 0,24 & 0.2 & 0,19 & 0,19 & 0,18 \\
\hline $\mathrm{MgO}$ & 736 & $7 A 4$ & $5 p 9$ & $4 z$ & 919 & 679 & 5,28 & 6,16 & 7,0 & $7, \mathbf{A}$ & 7,76 & 46 & 45 & 8,45 & 4,13 & 40 \\
\hline $\mathrm{Cm}$ & 10,00 & 8,7 & 9,30 & 7,33 & 11,45 & $9 / 4$ & 8,4 & 8,96 & 15,4 & $18, n$ & $12 \pi s$ & 97 & $9 / 5$ & 12,97 & 7,26 & 2,29 \\
\hline $\mathrm{KO}$ & 0,50 & 0,9 & 0,79 & 1,11 & 0,06 & 0,54 & 1,02 & 0,91 & 050 & 0,52 & 0,82 & 079 & 0,81 & 0,26 & 077 & 1,00 \\
\hline PyO & 0,26 & 0,2 & 0,59 & 0,85 & 0,14 & 0,72 & 0,31 & 0,00 & 0,12 & 0,14 & 0,2 & 0,31 & 0,8 & $0, \pi$ & 08 & 0A8 \\
\hline $\mathbf{P F}$ & 9,13 & 3,87 & 35 & 3,19 & 3,4 & 3,28 & 45 & 1,2 & $a, 0$ & 35 & $9 / 4$ & 234 & 2,20 & 1,1 & 207 & 1,9 \\
\hline Tot-1 & 9sse. & 99,4: & 100,0 & s, & $90 / 66$ & $9, y$ & 92 & 92 & 1005 & $\begin{array}{l}1607 \\
1\end{array}$ & 971 & $\$, \$$ & 10058 & 1005 & 100,51 & 101,a \\
\hline $\mathbf{Z n}_{\mathbf{n}}$ & 123 & 121 & 154 & 16 & $n$ & 182 & $1 \theta$ & 15 & 96 & $\boldsymbol{n}$ & 8 & no & 202 & 196 & 162 & 134 \\
\hline $\mathbf{M i}$ & 101 & 109 & $\pi$ & 85 & 74 & 118 & 62 & 0 & 115 & 94 & $\mathbf{1 1 2}$ & 64 & 99 & 51 & 69 & 46 \\
\hline $\mathrm{Cr}$ & 166 & 2 & 7 & 35 & 265 & 149 & 0 & 73 & 150 & 121 & 116 & 14 & 14 & 5 & 4 & 5 \\
\hline $\mathbf{v}$ & 356 & $m$ & 49 & 183 & 236 & 390 & 327 & 5 & 25 & 211 & 185 & 795 & $m 6$ & & 169 & \\
\hline Be & 2 & 1 & 2 & 2 & 1 & 2 & 2 & 2 & 1 & 1 & 1 & 2 & $1, \infty$ & 5 & 2 & 2 \\
\hline Cu & $\phi$ & 65 & 9 & $\infty$ & 77 & $\theta$ & 113 & Jot & $\pi$ & 30 & 3 & 120 & $12:$ & 24 & 49 & 29 \\
\hline $\mathbf{z}$ & 7 & 66 & 9 & 104 & 1 & 66 & 110 & $n$ & 61 & $n$ & 131 & 113 & 106 & 300 & 20 & 306 \\
\hline$\gamma$ & 92 & 36 & 4 & 9 & 16 & $\mathbf{x}$ & 44 & 3 & 16 & 16 & 15 & 0 & 3 & 46 & 50 & 0 \\
\hline$s$ & $x_{1}$ & Sat & 300 & 300 & 314 & 46 & 17 & 14 & 305 & 306 & 47 & 196 & 198 & $\Delta$ & 200 & 48 \\
\hline Be & 165 & 146 & 242 & 40 & 1326 & 167 & 20 & 316 & 123 & 284 & 130 & 26 & 278 & 62 & $7 \%$ & 64 \\
\hline$F \rightarrow O^{\circ}$ & 1275 & 19,14 & 1559 & 135 & 10,9 & 12,0 & & $\mathbf{I 6 ; \mathbf { A }}$ & 1056 & 10,12 & 9,5 & 16,60 & 16,96 & 10,02 & 1496 & 10,23 \\
\hline$F / F+M$ & 0,64 & 0,4 & 0,74 & 076 & 0,54 & 0,0 & $=$ & 0,7 & $0 \leq 0$ & ost & 0.5 & 070 & 08 & 05 & 038 & 0,2 \\
\hline
\end{tabular}

Lequende * Grupol Gropil * Grupo II 
Table 2 - REE data of Mafic Dikes of the Quadrilatero Ferrifero and Southern Espinhaço

\begin{tabular}{|c|c|c|c|c|c|c|c|c|c|c|c|}
\hline Samples, & La & ce & N1 & $3 \pi$ & Eu & Go & Oy & Ho & Er & th & U ل \\
\hline CAM2 & 11,52 & 29,07 & $\mathbf{t 5 , 8 7}$ & 3,65 & 1,123 & 3,281 & 3,807 & 0,746 & 1946 & 1,665 & 0,176 \\
\hline oAT1 & 8,164 & 23,29 & 12,56 & 2,601 & 0,628 & 2,148 & 2,147 & 0,415 & 1,05 & 0,876 & 0,099 \\
\hline OAM1 & 40,4 & 99,9 & 41,67 & 7,723 & 1,196 & 4,439 & 3,458 & 0,742 & 2252 & 2,689 & 0,376 \\
\hline 893 & 9,677 & 26,56 & 16,27 & 4,45 & 1,241 & 1366 & 4,85 & 0,98 & 2,692 & 2,282 & 0,27 , \\
\hline $\mathrm{PK}_{4}$ & 9,468 & 21,05 & 12,46 & 2,674 & 0,861 & 2,187 & 2,12 & 0,43 & 1,192 & 0,913 & 0,115 \\
\hline ITAII & 20,9 & 53,91 & 37.5 & 84 & 2,414 & 5,516 & 548 & $m$ & 2,2009 & 1,664 & 0.2 \\
\hline $\begin{array}{c}\text { RAR } \\
\text { II }\end{array}$ & 13,42 & 28 & 2,56 & 132 & 1,804 & 5,682 & 5,918 & 1,141 & 2,882 & 2,180 & 0,258 \\
\hline TAMU1] & 55,07 & 131,7 & 65,34 & 12,16 & 3,582 & 6.611 & 6.705 & 1,25 & 2,928 & 2,065 & 0,25 \\
\hline 71105 & 40,32 & 99,23 & 50,04 & 10,6 & 2,349 & 9,412 & 10,29 & 2,05 & 5,54 & 4,751 & $0,560^{\circ}$ \\
\hline DAt & $9 \mathrm{~g}, 35$ & 157,6 & 52,26 & 9,0112 & 1,966 & 7,298 & 7,01 & 1,41 & $\mathbf{3 , 6 7 7}$ & 3,499 & 0,455 \\
\hline
\end{tabular}

samples have $\mathrm{Ti} / \mathrm{Zr}$ ratios between 80 and 225, while in Group II the rations vary from 110 to 1227, and in Group III from 35 to 212. This indicates different degrees of mantle partial melting, but they may also be explained by mantle enriched or crustal contamination. The issue of enriched mantle versus crustal contamination has been widely discussed and, in most cases, its explanation has only been possible through isotope studies. The low ratios suggest enriched mantle sources, similar to those that originated the continental basalt flood, while the higher ratios may suggest a more depleted mantle.

In the $\mathrm{MgO} \times \mathrm{FeO}_{\mathrm{t}} \times \mathrm{Al}_{2} \mathrm{O}_{3}$ plot (Fig. 10), the samples fit to the field of continental rift-type basalts and therefore of continental intraplate regimes.

Rare Earth Elements (REE) Rare earth elements (REE) are important petrochemical discriminators for basic rocks. Even having similar chemical behaviour, the REE may be fracionated during melting and fractional crystallization (Henderson 1984), but may be a clue to source compositions. The mafic dike swarms of the Quadrilátero Ferrífero and Southeastern Espinhaço present three types of REE patterns, as shown in figure 11 .

Rocks of Group I are represented by two samples (Fig. 11). Sample DOI (quartz-chlorite schist), show LREE fractionation $[(\mathrm{La} / \mathrm{Sm}) \mathrm{N}=3,47]$ and HREE depletion $[(\mathrm{Dy} / \mathrm{Yb}) \mathrm{N}$ $=0,82]$. Sample IBI04 (gabbro), has a strong LREE fractionation $[(\mathrm{La} / \mathrm{Sm}) \mathrm{N}=6,39]$, but an almost flat HREE distribution $[(\mathrm{Dy} / \mathrm{Yb}) \mathrm{N}=1,28]$. The samples of Group II show a moderate LREE fractionation $[(\mathrm{La} / \mathrm{Sm}) \mathrm{N}=1,34-2,83]$ and a light to moderate HREE fractionation [( $\mathrm{La} / \mathrm{Sm}) \mathrm{N}=1,34$ to 2,08$]$. Group III has a LREE frationation $[(\mathrm{La} / \mathrm{Sm}) \mathrm{N}=2,53]$ and a less significant HREE fractionation ( $\mathrm{Dy} / \mathrm{Yb}) \mathrm{N}=1,47]$.

Spidergrams To construct the spidergrams we normalized the elements to primordial mantle values (Wood 1979). The elements are arranged, from left to right in decreasing order of incompatibility, thus providing information about the nature of the mantle source, crustal contamination and assimilation processes.

The classification of the mafic dikes into three distinct petrographic groups is also evidenced by their REE patterns. Both features corroborate the conclusions of Weaver \& Tarney (1981b) and Tarney \& Weaver (1987) that different magmatic pulses have different patterns. The different REE patterns in the same magmatic pulse may reflect an heterogeneous source (Condie et al. 1987).
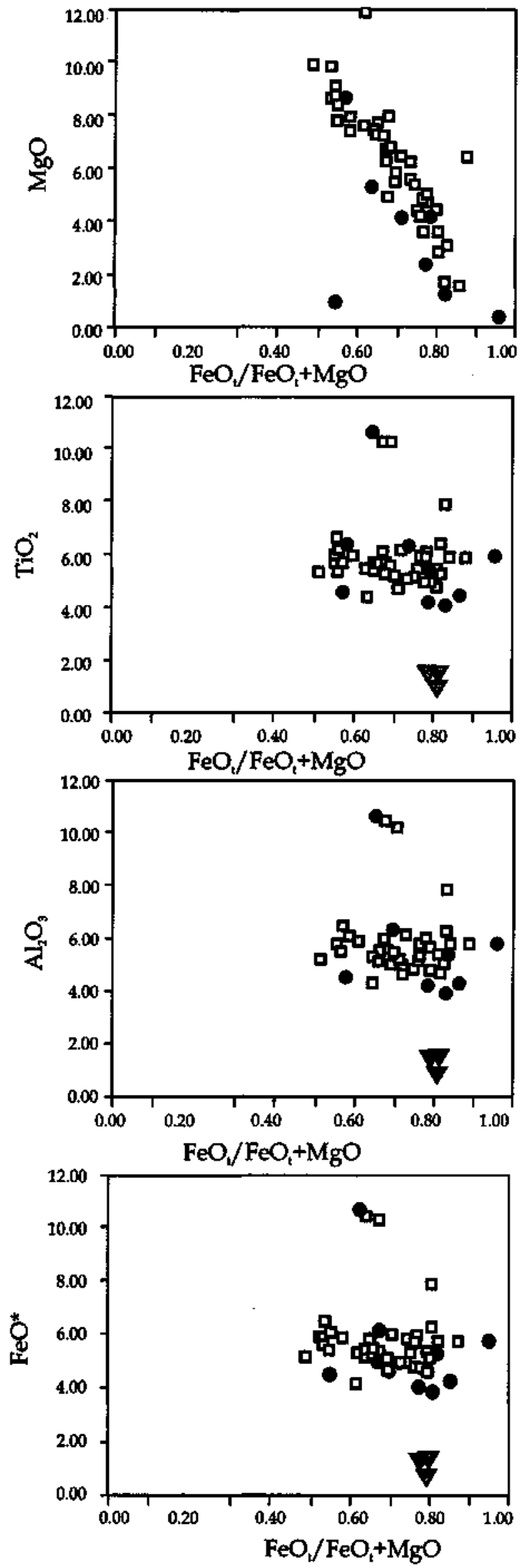

Figure 7 - Marker plots for $\mathrm{MgO}, \mathrm{Al}_{2} \mathrm{O}_{3}, \mathrm{TiO}_{2}$ and Feo versus the differentiation rate of the studied mafic rocks. 

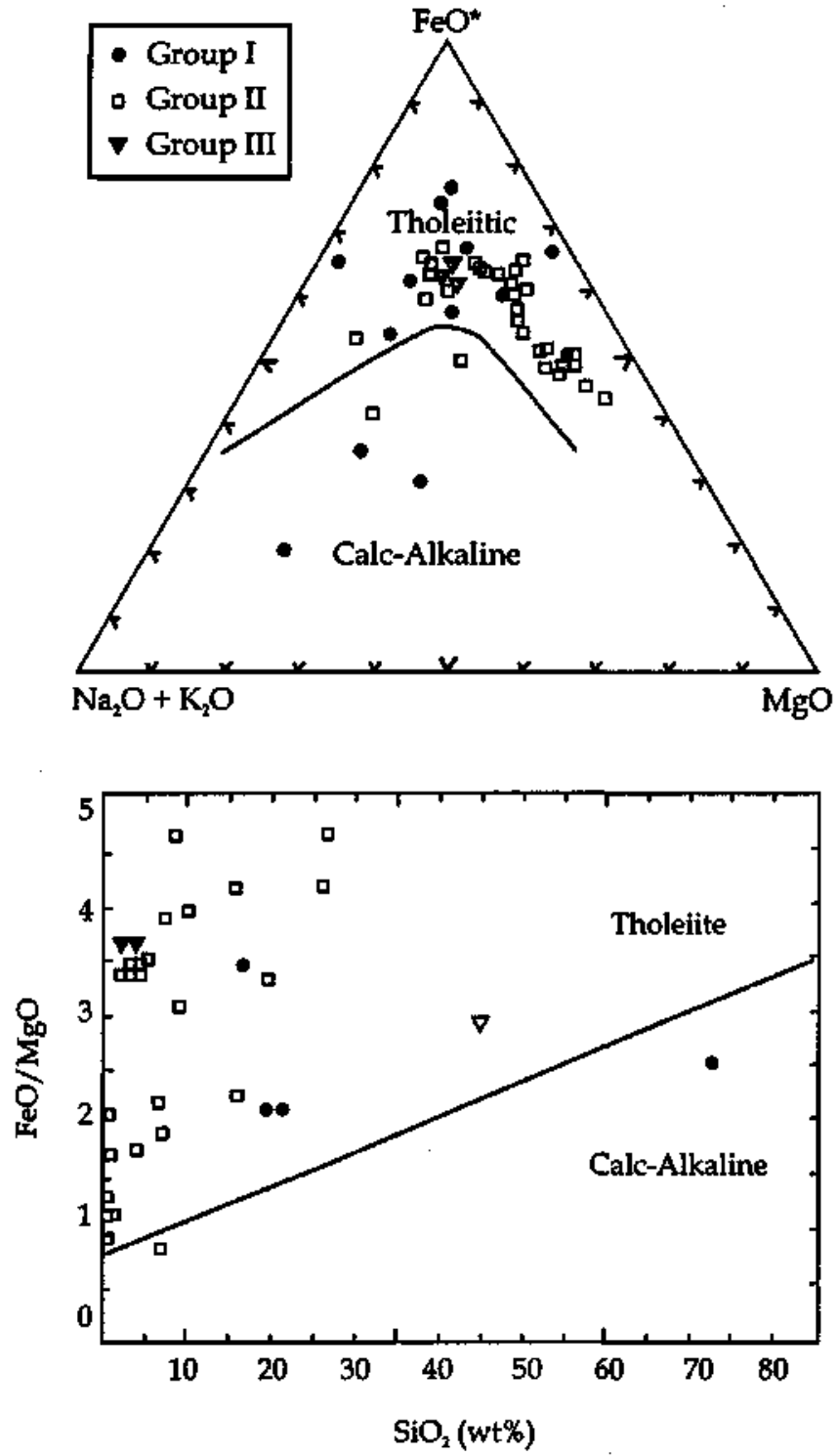

Figure $8-\mathrm{AFM}_{\mathrm{F}}$ and $\mathrm{SiO}_{2} \times \mathrm{FeO} / \mathrm{MgO}$ diagrams, respectively of Irvine \& Baragar (1971) and Miyashiro (1974) of the studied basic rocks.

Figure 12 contain the spidergrams of the studied mafic rocks, grouping the samples with similar behaviors. The diferent patterns corroborate a previous indication that the studied mafic dike swarms resulted from different magmatic pulses.

When normalized to primordial mantle, sample IBI04 of Group I has negative $\mathrm{K}$ and $\mathrm{Sr}$ anomalies, more accentuated in $\mathrm{Zr}$, as well as positive $\mathrm{La}, \mathrm{Ce}$, and $\mathrm{Nd}$ anomalies, and a slight positive $\mathrm{Sm}$ anomaly (Fig. 12a). The negative $\mathrm{K}$ and $\mathrm{Zr}$ anomalies are, possibly, due to crustal contamination/assimilation. Sample D01 has negative Sr, P, and Ti anomalies and positive $\mathrm{K}, \mathrm{Nd}$ and $\mathrm{Zr}$ anomalies. The negative $\mathrm{P}$ and $\mathrm{Ti}$ anomalies can be explained either by a source poor in these elements, or by retention in the mantle during partial melting. The positive $\mathrm{K}$ and $\mathrm{Zr}$ anomalies are, possibly, due to metamorphism. The positive $\mathrm{Zr}$ anomaly may also be explained by contamination with rocks of the upper crust, as proposed by Taylor \& McLennan (1985).
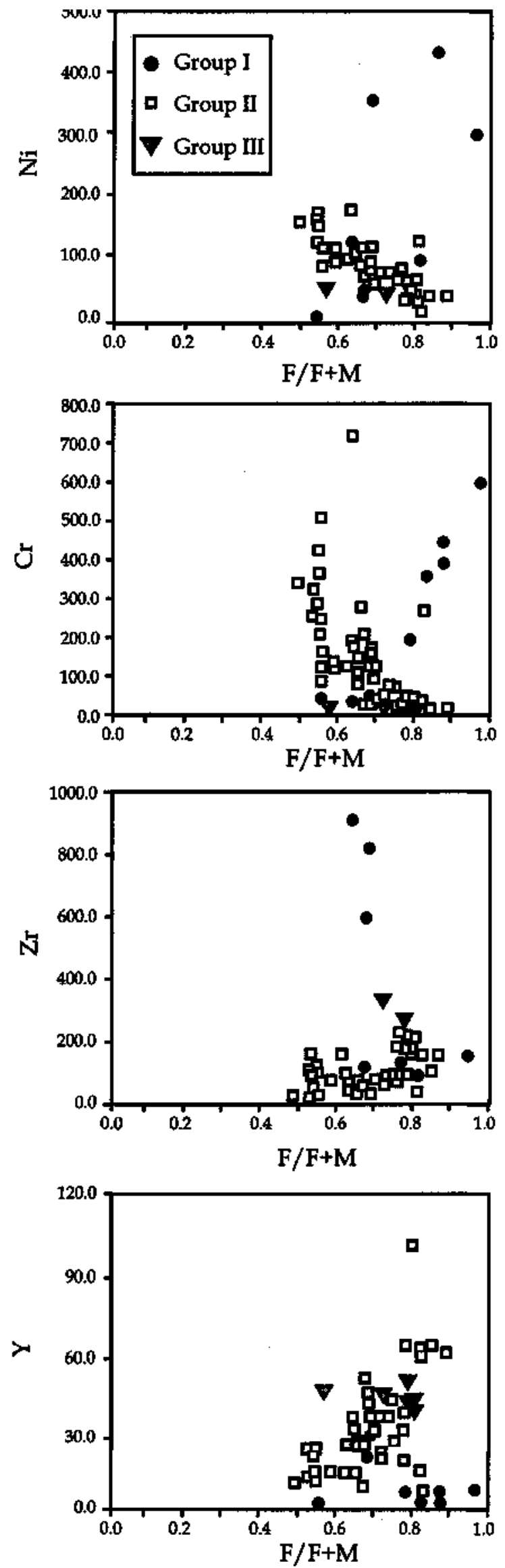

Figure 9 - Ni, Cr, Zr, and Y versus differentiation index of the studied basic rocks 


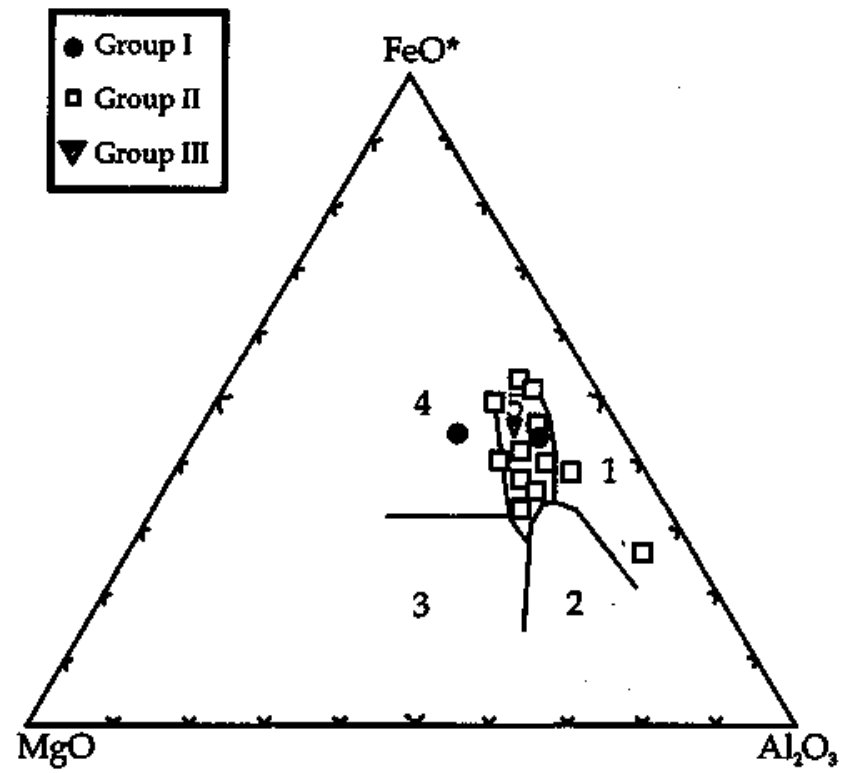

1 - Spreading Center Island; 2 - Orogenic; 3 - Ocean Ridge and Floor; 4 - Ocean Island; 5 - Continental

Figure 10 - $\mathrm{MgO} \times \mathrm{FeO}_{t} \times \mathrm{Al}_{2} \mathrm{O}_{3}$ tectonic setting diagram of Pearce et al. (1977)

Samples of Group II (Fig. 12b) shows small negative Sr, $\mathrm{Zr}$, and occasionally also $\mathrm{Ba}$ anomalies, and positive $\mathrm{Sm}$, eventually also Ti anomalies. Sample TIM05 has negative K, $\mathrm{Sr}, \mathrm{Zr}$ and positive Ti anomalies. Sample SB03 has small negative $\mathrm{Ba}$ and $\mathrm{K}$, negative $\mathrm{Sr}$ and $\mathrm{Zr}$, and positive $\mathrm{Sm}$ anomalies. Despite of these differences, these patterns may be grouped into one single envelope. Sample PK04 differs from others of the same group by its positive $\mathrm{Sr}$ and $\mathrm{Zr}$ anomalies, and sample SJC02 (Fig. 12c) by its slight negative B a and Sr, and a strong positive $\mathrm{P}$ and less strong $\mathrm{Sm}$ anomalies.

Some samples of Group II have patterns that are similar to tholeiitic island arc basalts, while others are more compatible with continental rift basalts. The negative $\mathrm{Zr}$ anomalies may be due to retention in the mantle residual melt.

Group III has negative $\mathrm{K}, \mathrm{Sr}$ and $\mathrm{Zr}$, and positive $\mathrm{La}, \mathrm{Ce}$ and $\mathrm{Nd}$ anomalies (Fig. 12d). The La and Ce enrichment can be explained either by their mobility during metamorphism and/or hydrothermal alteration, or by a larger fractionation of these elements in the source, or even by crustal contamination. The positive $\mathrm{P}$ anomaly may be due to crustal contamination.

The negative $\mathrm{Sr}$ anomalies of these groups probably reflect its mobility during metamorphism and /or the hydrothermal alteration. However, part of these anomalies may also be due to pyroxene rather than plagioclase fractionation.

Radiometric Data Previous radiometric datings of the studied mafic dikes have been published by Teixeira (1995), Machado et al. (1989b), and Machado \& Carneiro (1992). More recently we obtained complementary data.

By means of K/Ar ages, Teixeira (1995) attempts to estabilish the main extension period of the southern portion of the São Francisco Craton. The authors suggests that the more important crustal fracturing events took place by $2,1-1,85 \mathrm{Ga}$ (K/Ar in amphibole), and 1,7-1,5 Ga (K/Ar in total rock, amphibole and plagioclase), and, therefore, that the most important mafic dike intrusions in the craton ocurred during the Paleo-Mesoproterozoic times.

The most important mafic dike swarm of Group II cut the basement rocks of the Southeastern Espinhaço region, these
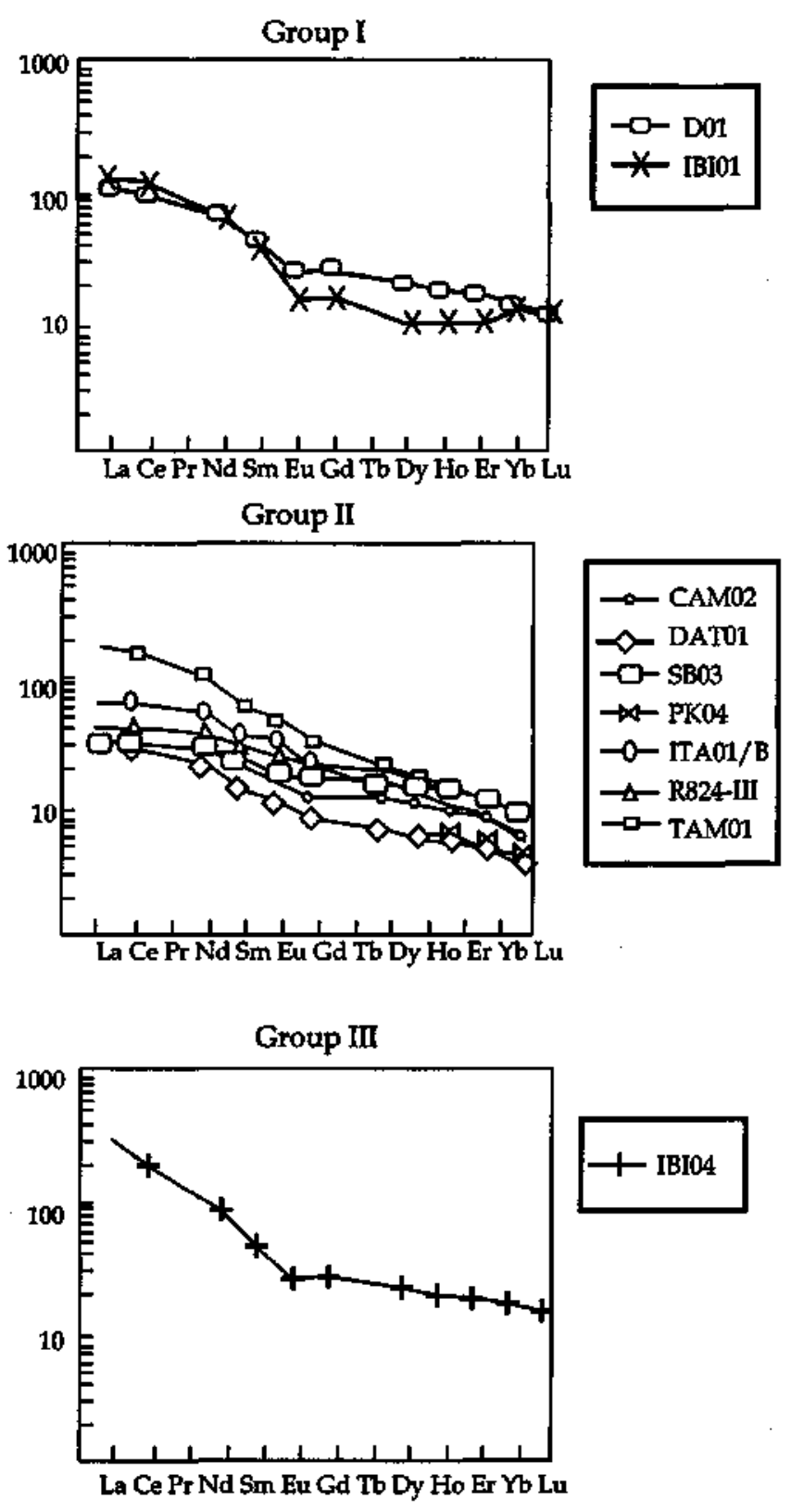

Figure 11-REE patterns of the three mafic dike swarms of the
Quadrilatero Ferrffero and Southern Espinhaço

dikes have zircon and baddeleyite with an $\mathrm{U} / \mathrm{Pb}$ age of 906 +/ -2 Ma (Machado et al. 1989b). A similar K/Ar age of 1,0 Ga was also found by Machado \& Carneiro (1992) in green hornblende of rocks belonging to Group II of the Quadrilátero Ferrífero.

For this paper we dated baddelyite of the Ibirite gabbro (IBI04) at the Royal Ontario Museum. The extraction of U and $\mathrm{Pb}$ used a procedure described elsewhere (e.g. Heaman \& Machado 1992). The isotopic composition of these elements was determined on a VG354 mass spectrometer in single collector mode. The $\mathrm{U}$ and $\mathrm{Pb}$ blanks are estimated to be 0.5 and $2.0 \mathrm{pg}$, respectively. These values must be accurate, considering that total common $\mathrm{Pb}$ in fraction $\# 2$ is of $3.1 \mathrm{pg}$. The used U decay constants are those of Jaffey et al. (1971). The discórdia calculation used the program of Davis (1982).

Sample IBI-04 contained a small number of tinny $(<\mu)$ baddeleyite crystals. The baddeleyite yield was insufficient to 
Table 3 - U-Pb results for baddeleyite of sample IBI-04.

\begin{tabular}{|c|c|c|}
\hline Fraction & $f 1$ & $n$ \\
\hline Weion ImitroarmsI & $\mathbf{1}$ & O \\
\hline U (ppm) & Bg1 & 50 \\
\hline$P_{0}$ lopmonl & 200 & 50 \\
\hline Th lpponl & 46 & 92 \\
\hline Total Cormmon fo lpal & $8 . ?$ & 3.1 \\
\hline${ }^{310} \mathrm{Pt}^{20 x} \mathrm{Pt}$ imeoured & 2,468 & 24,273 \\
\hline${ }^{200} p_{0} x^{2 x}=1$ & $0,255600+78$ & $0.10212+18$ \\
\hline${ }^{203} \mathrm{P}^{200} \mathrm{U}$ & $4.2547+115$ & $0.8638+15$ \\
\hline $\mathrm{mp}_{\mathrm{Pb}}^{\mathrm{j}^{30}}$ & 0.1BA32+8 & $0.06135+7$ \\
\hline 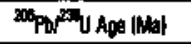 & 1671 & 627 \\
\hline 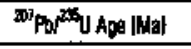 & 1885 & 602 \\
\hline 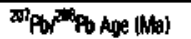 & 1702 & 652 \\
\hline
\end{tabular}

(a)

(c)

\section{Group I}

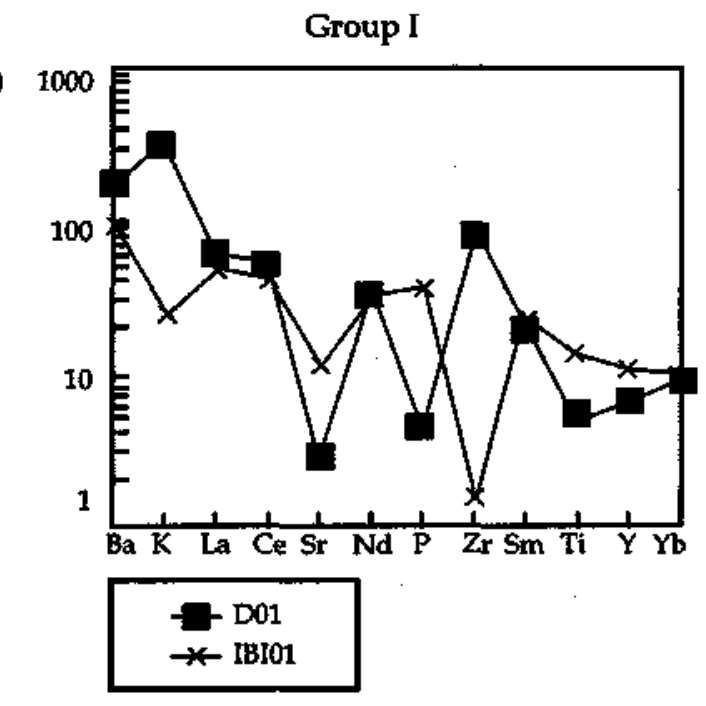

Group II

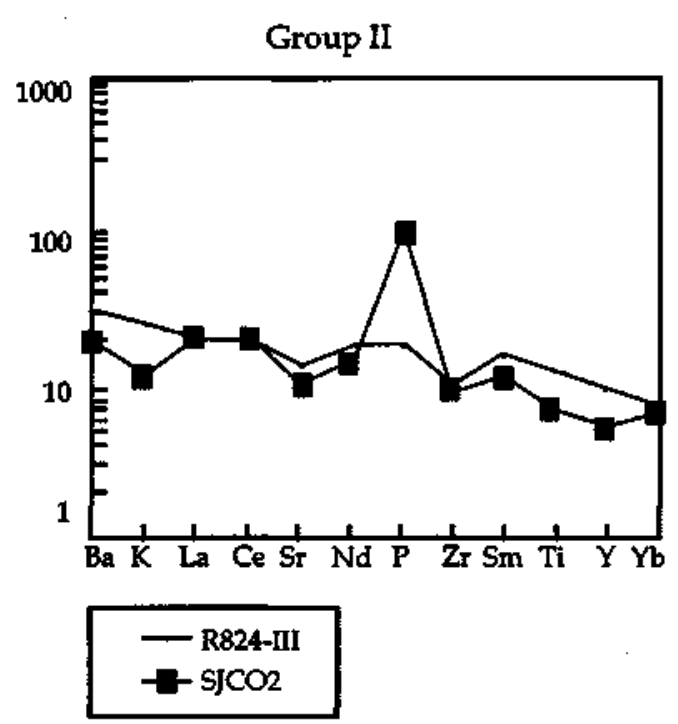

analyse more than two fractions. The grains were then subivided into a smaller fraction of best transparent fragments (\#1 in Table 3) and a larger fraction of fractured grains. Many grains were frosted, probably due to incipient formation of zircon by metamorphism. The two fractions have contrasting ${ }^{207} \mathrm{pb} /{ }^{206} \mathrm{pb}$ ages of 1702 and $652 \mathrm{Ma}$, atypical for pristine

baddeleyite. The best quality baddeleyite grains (\#1) have the oldest age, interpreted to closely approximate the time of the gabbro emplacement. The poor quality baddeleyite grains contain some metamorphic zircon and may more closely reflect the time of the gabbro metamorphism.

A discórdia line (Fig. 13) of the two analyses yield an upper intercept age of $1714 \pm 5 \mathrm{Ma}$, interpreted as the best estimated for the time of gabbro emplacement while the lower intercept age of $618 \pm 3 \mathrm{Ma}$ is interpreted as the time of gabbro metamorphism. The lower uranium content $(503 \mathrm{ppm})$ of the poorer quality fraction (\#2) is consistent with metamorphic zircon which, in other examples of coronitic gabbros (Davidson \& Van Breemen 1988, Heaman \& Le Cheminant 1993) tends to relatively low $\mathrm{U}$ contents $(<150 \mathrm{ppm})$. If this interpretation is correct, then the amount of discordance along the discórdia line for fraction \#2 cannot be explained only by the

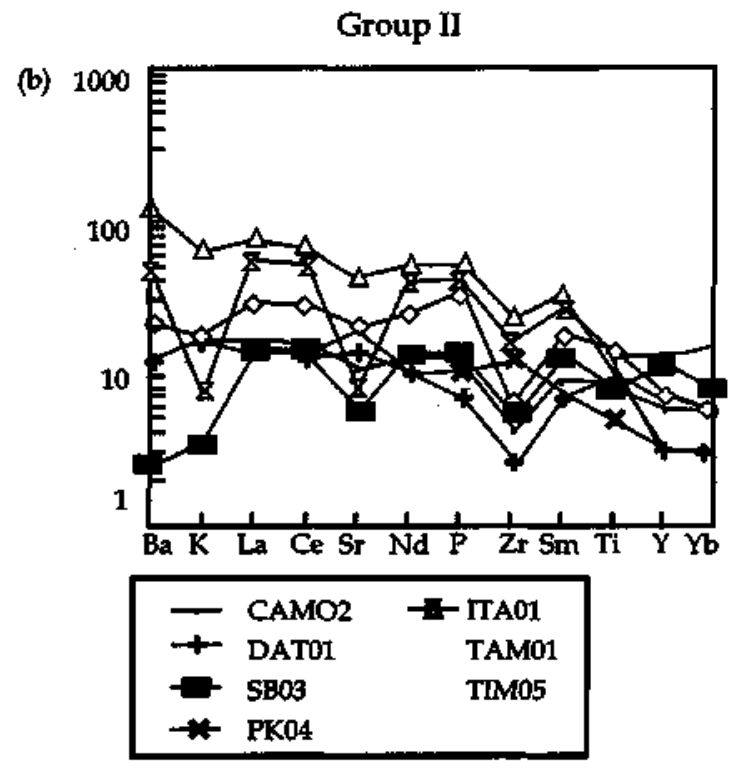

Group III

(d)

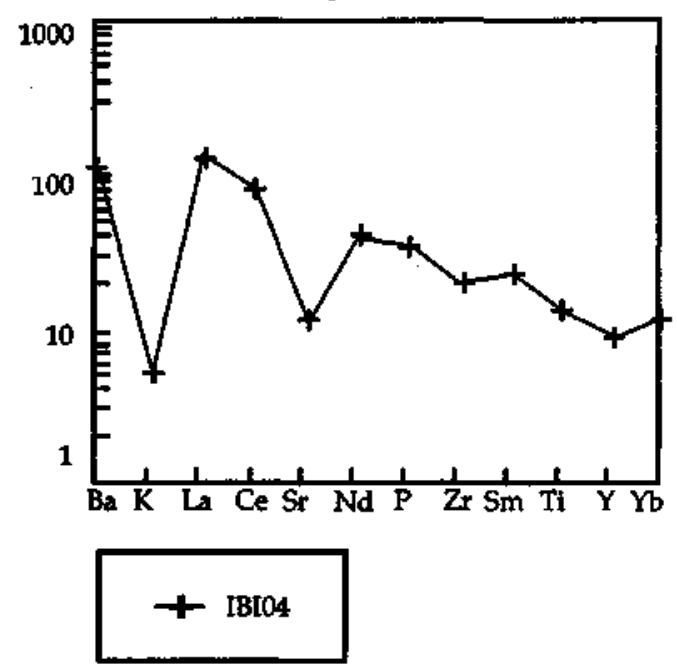

Figure 12 - Spidergram of the three mafic dikes swarms of the Quadrilátero Ferrifero and Southeastern Espinhaço 
presence of some metamorphic zircon and U mass balance considerations. The baddeleyite in this analysis must also have experienced significant $\mathrm{Pb}$-loss, as documented in in few rare examples (e.g. Heaman \& Grotzinger 1992).

Of Group III, only the sample C088, a diabase of the Quadrilátero Ferrífero, was dated, yielding a K/Ar whole rock age of $120 \mathrm{Ma}$, thus correlated to the early stages of Gondwanaland breakup (Chemale et al. 1991, Silva et al. 1991 a and $\mathrm{b}$ ). This age is compatible with the K/Ar whole-rock 170 to $220 \mathrm{Ma}$ interval previously obtained by Dossin et al. (1995) in rocks of the same group.

Two samples were processed for Sm-Nd dating (SB03 e DAT01) at the Instituto de Geociências of the University of São Paulo. The experimental procedure includes the technique of acid digestion, separation of $\mathrm{Sm} / \mathrm{Nd}$ by using a small column of ion exchange, and isotope ratio measurements in a thermal ionization mass spectrometer with multiple and single

Table 4 - Sm-Nd results for Mafic Dikes of the quadrilátero Ferrifero and Southeastern Espinhaço

\begin{tabular}{|c|c|c|c|c|c|c|}
\hline & $\sin$ & Nd & ${ }^{w_{\text {Sinl }}}$ & ith & tid & $\underset{\text { IGo] }}{\mathbf{I}_{0}}$ \\
\hline $\operatorname{sit}_{1 \mathrm{H}} \mid \mathrm{Gr}$ & $\begin{array}{c}5.157 \\
+1 \\
0.004\end{array}$ & $\begin{array}{c}16.39+1- \\
0.00 \%\end{array}$ & $\begin{array}{c}0.1707 \\
+\% \\
0.00015\end{array}$ & $\begin{array}{c}0.512821 \\
+1 . \\
0000027\end{array}$ & $+1,34$ & 259 \\
\hline $\begin{array}{l}\text { DATO1 } \\
\text { IGI. III }\end{array}$ & 3.15 & 14.39 & $\begin{array}{c}0.130 \\
+1 \\
0.00999\end{array}$ & $\begin{array}{c}0.5+2325 \\
+\% \\
0.00124\end{array}$ & 3.20 & 1.35 \\
\hline
\end{tabular}

collector systems (Sato et al. unpublished).

The Sm-Nd data indicate end (T) $(+1,34$ and -3.20$)$ and model ages $\left(\mathrm{T}_{\mathrm{DM}}\right)$ of ca. 2.59 and $1.35 \mathrm{Ga}$. The results are presented in Table 4.

The analytical data indicate a $\mathrm{Nd}$ isotopic composition depleted in ${ }^{143} \mathrm{Nd}$. The negative epsilon values indicate that

the basic rocks derived from sources with lower $\mathrm{Sm} / \mathrm{Nd}$ ratio than the condritic reservoir. This means that such rocks derived from, or assimilated old crustal sources lowered in the $\mathrm{Sm} / \mathrm{Nd}$ ratio when their magmas separated from CHUR.

The isotopic data, the high LIL content, and the quartz-normative nature of these dikes suggest crustal contamination during intrusion. Nd isotopic characteristics of the both studied areas suggest long crustal residence of the dikes precursor magmas.

GEOTECTONIC CONTEXT OF THE MAFIC DIKE SWARMS From the above described data, it is possible to infer the geotectonic context of the mafic dike swarms of the southern portion of the São Francisco craton and deduce their stratigraphic relationship with the supracrustals rocks. The data indicate that the Minas and Espinhaço supracrustals were intruded by at least three basic dike swarm events.

The first event, with a north-south direction, cuts sin-rift sections of the Espinhaço Supergroup and has been correlated to the opening of the Espinhaço sedimentary basin (Silva et al. 1991a and b). Metarhyolites associated to the early stages of sedimentation yield a zircon $\mathrm{U} / \mathrm{Pb}$ age of $1,7 \mathrm{Ga}$ (Brito Neves 1979), thus having the same age of the basic dikes.

These data show that the basic dikes may correspond to the first magmatic pulse that cuts through the Paleoproterozoic supracrustals of the studied regions. They also indicate that these rocks formed during the extensional event responsible for the Espinhaço basin. On the other hand, the occurrence of felsic rocks of the same age as that of the basic rocks points
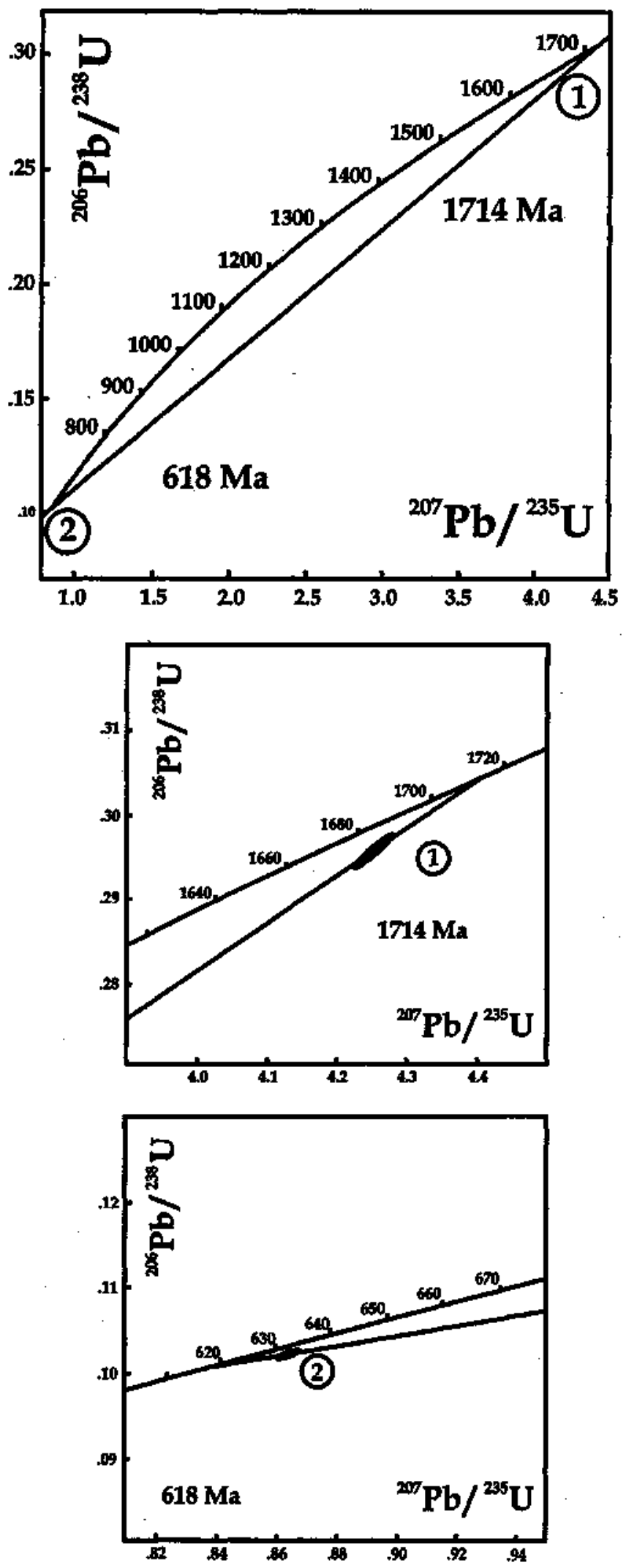

Figure 13 - Baddelyite U/Pb discórdia line of sample IBI04

to a bimodal magmatism (Silva et al. 1995), respectivelly from lower crustal and mantelic sources.

Dike rocks of the second pulse ( $906 \pm 2 \mathrm{Ma}$ - Machado et al. 1989b) are deformed and metamorphosed under green- 
schist facies, but with preserved igneous features, and cut through all units of the Minas and Espinhaço Supergroups. According to Machado et al. (1989b) this pulse of mafic dikes preceedes the beginning of the sedimentation of the São Francisco Supergroup. Recently, the Espinhaço Project (1994) evidenced that they also intruded rocks of the Macaubas Group.

The second pulse marks an important period of crustal extension of the Brasiliano-Panafrican cycle early stages. Mafic rocks of this event also been reported ensewhere in Brazil and in the Kalahari craton (D' Agrella-Filho et al. 1990, Renne et al 199, Heaman 1991).

$\mathrm{Sm} / \mathrm{Nd}$ data of the metagabbros of this group have a $\mathrm{ENd}$ (T) $\left(+1,34\right.$ and - 3.20) and model ages $\left(\mathrm{T}_{\mathrm{DM}}\right)$ of ca. 2.59 and $1.35 \mathrm{Ga}$. They also have a high content in LIL and normative quartz; This may suggest crustal contamination, and indicate that magmas of this group resulted from partial melting of an enriched sub-continental lithosphere, further modified by their interaction with the continental crust.

Group III is the youngest in the studied regions and consists of undeformed and unmetamorphosed diabase dikes that cut through all Precambrian structures. Their K/Ar age is of ca. $120 \mathrm{Ma}$ (Chemale Jr. et al. 1991, Silva et al. 1991a and b), thus correctable with the extensive flood basalts of the Paraná Basin, in southern Brazil. The few geochemical data suggest that the Neoproterozoic gabbros and Mesozoic dikes are similar, as previously reported by Oliveira \& Tarney (1990). These similiralities indicate that the mantle composition did not change significantly from Proterozoic to the Mesofanerozoic, as also suggested by Mazzucchelli et al. (1995) for the mafic dikes in Uruguay.

CONCLUSIONS Despite of still being fragmentai, the geological knowledge of mafic dikes of the southeastern Minas Gerais improved in the last few years. The available data indicate that the supracrustal rocks of Minas and Espinhaço Supergroups were intruded by at least three basic magmatic events, each with its own structural, petrographical, geochemical, and radiometric characteristics.

The first magmatic pulse is related to the opening of the Espinhaço sedimentary basin, at about 1.7-1.5 Ga. The second is associated with the Panafrican-Brasiliano event and has an age of $906+/-2 \mathrm{Ma}$. The third has an age of approximately 120 Ma and took place during the fragmentation of Gondwana. This leds to the conclusion that the São Francisco Craton underwent successive periods of crustal extension, the most voluminous and widespread taking place during the Neoproterozoic Panafrican-Brasiliano orogeny.

Acknowledgements To Dr. Márcio Martins Pimentel, University of Brasilia, for suggestions and critical comments on $\mathrm{U} / \mathrm{Pb}$ and $\mathrm{Sm} / \mathrm{Nd}$ data. To Dr. Colombo Tassinari and the staff of the Centro de Pesquisas Geocronológicas, Instituto de Geociências/University of São Paulo, for the $\mathrm{Sm} / \mathrm{Nd}$ analyses. This research was partially supported by Conselho Nacional de Desenvolvimento Tecnológico e Científico (CNPq) and UFOP/Petrobrás/ Fundação Gorceix Agreement.

\section{REFERENCES}

BRITO NEVES, B.B; KAWASHITA, K. \& DELHAL, J. 1979. A evolução geocronológica da Cordilheira do Espinhaço: Novos Dados e integração. Rev. Bras. Geoc., 9(1):71-85.

CHEMALE JR., R; ROSIÈRE, C.A. \& ENDO, I. 1991. Evolução tectônica do Quadrilátero Ferrífero, Minas Gerais -Um modelo (Tectonic Evolution of the Qua- drilátero Ferrífero, Minas Gerais. A Model). Pesquisas, 18: 104-127.

CHEMALE JR., F.; ROSIÈRE, C.A. \& ENDO, I. 1994. Tectonic Evolution of the Quadrilátero Ferrífero, MG, Brazil. Precam. Res., 65:25-54.

CONDIE, K.C. 1985a. Secular Variation in the Composition of Basalts: an index to mantle evolution. Jour. Petrology, 26:545-563.

CONDIE, K.C.; BOBROW, D.J. \& CARD, K.D. 1987. Geochemistry of Precambrian Mafic Dike from Southern Superior Province. In: Halls, H.C. and Fahrig, W.F. (eds.) Mafic Dike Swarms. Geol. Assoc. of Canada Special Paper, 34:95-108.

CONDIE, K.C. \& HUNTER, D.R. 1976. Trace element geochemistry of archean granitic rocks from the Barbeton region, South Africa. Earth Plan. Sci. Lett. 29:389-400.

CORDANI.U.G.; KAWASHITA.K; MUELLER.G.; QUADE.H.; REIMER.V. \& ROESER.H.P. 1980. Interpretação tectônica e petrológica de dados geocronológicos do embasamento do bordo sudeste do Quadrilátero Ferrífero, Minas Gerais. Acad. Brás. Cienc., Anais...Rio de Janeiro, 52:785-799.

CORDANI,U.G.; TEIXEIRA.W. \& SIGA JR.,O. 1989. Geocronologia do Quadrilátero Ferrífero. In: XXI Semana de Estudos de Ouro Preto, SICEG, Ouro Preto, Boi. 21:27-44.

D'AGRELLA-FILHO, M.S.; PACCA, I.G.; RENNE, P.R.; ONSTOTT, T.C. \& TEIXEIRA, W. 1990. Paleomagnetism of Middle Proterozoic $(1,01$ to $1,08 \mathrm{Ga}$ ) mafic dikes in southeastern Bahia State - São Francisco Craton, Brazil. Earth Plan. Sci. Lett., 101:332- 348.

DAVIS, D.W. 1982. Optimum linear regression and error estimation applied to U/Pb data. Can. J. Earth Sci. 19: 2141-2149.

DAVISON, A. \& VAN BREEMEN, O. 1988. Baddeleyite-zircon relationships in coronotic metagabbros, Greenville Province, Ontario: implications for geochronology. Contrib. Mineral. Petrol., 100:291299.

DORR, J.N. 1969. Physiografic, stratigraphic, and structural development of the Quadrilátero Ferrífero, Minas Gerais. U.S. Geol. Surv. Pap., 641A-.110S; Washington.
DOSSIN,T.M.;DOSSIN, I. A.; CHAR VET, J. \& BONHOMME, M.G. 1995. $\mathrm{K}-\mathrm{Ar}$ chronology of a Mesozoic dike swarm from southern Espinhaço Region (SE Brazil). Jour, of South Am. Ear. Sci., 8:47-53.

FRIZ-TOPPER, A. 1991. Geochemical characterization of Pan-African dike swarms in Southern Sinai: from continental margin to intraplate magmatism. Precam. Res., 49:281-300.

HALLS, H. 1982 . The Importance and Potencial of Mafic Dikes Swarms in Studies of Geodynamic Process. Geoscience Canada, 9:145-154.

HEAMAN, L.M.1991. U-Pb dating of giant radiating dike swarms: potential for global correlation of mafic magmatic events. In:Teixeira,W., Ernesto, M. and Oliveira, E.P.eds. Ill INTERN. Symposium on Mafic Dikes. Extends Abstracts, p.7-8. São Paulo.

HEAMAN, L.M. \& GROTZINGER, J.P. 1992. 1.08 G.a. diabase sills in the Pahrump Group, California. Implications for development of the Cordilleran miogeocline. Geology, 20:637-640.

HEAMAN, L.M \& MACHADO, N. 1992. Timing and origin of midcontinent rift alkaline magmatism, North America: evidence from Coldwell Complex. Contrib. Mineral. Petrol., 110:289-303.

HEAMAN, L.M \& LECHEMINANT, A.N. 1993. Paragenesis and U-Pb systematics of baddeleyite (ZrOa). Chemical Geology (Including Isotope Geoscience), 110:95-126.

HENDERSON, P. 1984. Rare Earth Element Geochemistry Developments in Geochemistry, 2. Elsevier, New York, 51 Op.

IRVINE, I.N. \& BARAGAR, W.R.A. 1971. A guide to the chemical classification of the common volcanic rocks. Can. Jour. Earth Sci., 8: $523-548$.

JAFFEY, A.H., FLYNN, K.F., GLENDENIN, L.E., BENTLEY, W.C. \& ESSLING, A.M. 1971. Precision measurement of half-lives and specific activities of ${ }^{235} \mathrm{U}$ and ${ }^{238} \mathrm{U}$. Phys. Rev., 4:1889-1906.

LADEIRA, E. A. 1980. Metallogenesis of Gold at the Morro Velho Mine, and in Nova Lima District, Quadrilátero Ferrifero, Minas Gerais, Brazil. Unpubl. PhD thesis, University of Western Ontario, London, 272pp.

LE MAITRE, R.W. 1976. A proposal by the IUGS Subcomission on the Systematics of Igneous Rocks for a Chemical classification of volcanic rocks based on the total alkali silica (TAS) diagram. Australian Jour. Earth Sci., 31: 243-255.

LINDSLEY.D.H.1983. Pyroxene thermometry. Am. Mineral., 68:477-493. 
MACHADO, N.; NOCE, C.M.; BELO DE OLIVEIRA, O.A. \& LADEIRA, E.A. 1989a. Evolução geológica do Quadrilátero Ferrífero Arqueano e Proterozóico Inferior, com base em geocronologia U/Pb. In: $5^{\circ} \mathrm{SIMP}$. GEOL. NÚCLEO MG - $1^{\circ}$ SIMP. GEOL. Núcleo Brasília, SBG NÚCLEO MG, Anais...Belo Horizonte, BOL. 10:1-5.

MACHADO, N.; SCHRANK, A.; ABREU, F.R.DE; KNAUER, L.G. \& ALMEIDA-ABREU, P.A. 1989b. Resultados o preliminares da geocronologia $\mathrm{U} / \mathrm{Pb}$ na Serra do Espinhaço Meridional.In: $5^{\circ} \mathrm{SIMP}$. GEOL., Núcleo de MG - $1^{\circ}$ SIMP. GEOL. Núcleo Brasília, SBG Núcleo MG, Anais. ..Belo Horizonte, BOL. 10: 171 -174.

MACHADO, N. \& CARNEIRO, M.A. 1992. U-Pb evidence of late Archaean tectono- thermal activity in the southern São Francisco shield, Brazil. Can. J. Earth Sci., 29(11):2341- 2346.

MARSHAK, S. \& ALKMIM, F.F. 1989. Proterozoic extension/contraction tectonics of the São Francisco Craton of the São Francisco Craton and adjacent regions, Minas Gerais, Brazil: a kinematic model relating Quadrilátero Ferrífero, São Francisco Basin and Cordilheira do Espmhaco.Tectonics, 8 (3):555-571.

MAZZUCCHELLI, M.; RIVALENTI, G.; PICCIRILLO.E.M.; GIRARDI,V.A.V.; CIVETTA, L. \& PETRINI.R. 1995. Petrology of the Proterozoic mafic dike swarm of Uruguay and constraints on their mantle source composition. Precam. Res., 74:177-194.

MIYASHIRO, A. 1974. Volcanic rock series in island arcs and active continental margins. Am. J. Sci. 274:321-355.

NAKAMURA, N. 1974. Determination of REE, Ba, Fe, $\mathrm{Mg}, \mathrm{Na}$ and $\mathrm{K}$ in carbonaceous and ordinary chondrites. Geochem. Cosmoche. Acta. 38: 757-775.

OLIVEIRA, E.P. \& TARNEY, J. 1990. Petrogenesis of the Canindé de São Francisco Complex: a major Late Proterozoic gabbroic body in the Sergipe Foldbelt, northeastern Brazil. Jour. South Am. Earth Sci., 3:125-140.

PEARCE, J.A.; GORMAN, B.E. \& BISKETT, T.C. 1977. The relationship between major elements chemistry and tectonic environment of basic and intermediate volcanic rocks. Earth Planet. Sci. Lett., 36:121-132.

PFLUG, R. \& RENGER, F. 1973. Estratigrafia e evolução geológica da margem sudeste do cráton Sanfranscicano. In: XXVII CONGR. BRAS. GEOL., Anaw...Aracaju, 2:5-19.

POLDER V AART, A. \& HESS.H.H. 1951. Nomenclature of clinopyroxenes in the system $\mathrm{CaMgSI}_{2} \mathrm{O}_{6}-\mathrm{CaFeSI}{ }_{2} \mathrm{O} 6-\mathrm{Mg}_{2} \mathrm{SI}_{2} \mathrm{O}_{6}-\mathrm{FeS} \mathrm{i}_{2} \mathrm{O}_{6}$. Journ. Geol., 59:472.

RENNE, P.R ; ONSTOTT, T.C ; D'AGRELLA-FILHO, MS ; PACCA, IG. \& TEIXEIRA, W. $1990 .{ }^{40} \mathrm{Arr}^{39} \mathrm{Ar}$ dating of $1,0-1,1$ Ga magnetizations from the São Francisco and Kalahari cratons: tectonic implications for Pan-African and Brasiliano mobile belts. Earth Plan. Sci. Lett., 101:349-366.
SATO, K., TASSINARI, C.C.G., KAWASHITA.K \& PETRONILHO, L. Método geocronológico Sm/Nd no IG-USP e suas aplicações (unpublished).

SCHORSCHER, H.D. 1978. Komatiitos na estrutura greenstone belt Série Rio das Velhas. Quadrilátero Ferrífero, Minas Gerais, Brasil. XXX Congr. Bras. Geol., Anais...Recife, Bol. Esp. 1:292-293.

SCHORSCHER, H.D., CARBONARI, F.C., POLONNA, J.C. \& MOREIRA J.M.P. 1982. Quadrilátero Ferrífero - Minas Gerais State: Rio das Velhas greenstone belt and Proterozoic rocks. Intern. Symp. Early Arch. Prot., Salvador, Excur. Annex., 46 pp.

SILVA, A.M.; KUYUMJIAN, R.M. \& CHEMALE JR., F. 1991a. Mafic dikes swarms in the southern São Francisco Craton, Southeastern Brazil. In:Teixeira,W., Ernesto.M. and Oliveira,E.P.eds. Ill Intern. Symposium on Mafic Dikes. Extend. Abstracts, p.90- 93. São Paulo.

SILVA, A.M.; KUYUMJIAN, R.M. \& CHEMALE JR., F 1991b. Rochas básicas do Quadrilátero Ferrífero e Espinhaço Meridional: Estudos preliminares. REM - Revista da Escola de Minas, Ouro Preto, $45(1 \mathrm{e}$ 2): $60-62$.

SILVA, A. M.; CHEMALE JR., F. \& HEAMAN.L. 1995. The Ibirité Gabbro and The Borrachudo Granite: the rift-related magmatism of Mesoproterozoic age in the Quadrilátero Ferrífero, MG. Anais ... $8^{\circ}$ Simpósio de Geologia de Minas Gerais, Diamantina, MG: 89-90.

TAYLOR, S.R. \& MCLENNAM, S.M. 1985. The Continental Crust:its Composition and Evolution. Blackwell, Oxford, 312pp.

TARNEY, J \& WEAVER, B.L. 1987. Geochemistry and Petrogenesis of Early Proterozoic Dike Swarms. In: Hall, H.C. and Fahrig, W.H. (eds.) Mafic Dike Swarms. Geol. Assoc. of Canada Special Paper 34, p. 81-94.

TEIXEIRA, W. 1985. A evolução geotectônica da porção meridional do cráton São Francisco, com base em interpretações geocronológicas. $\mathrm{PhD}$ Thesis, Unpubl., IG-USP, São Paulo.

THOMPSON, R.N.; MORRISON, M.A.; HENRY, G.L. \& PARRY, S.J. 1984. An assessment of the relative roles of crust and mantle in magma genesis: An elemental approach. Philos. Trans. Royal Soc. London A310:549-590.

ULHEIN, A.; TROMPETTE, R.R. \& EGYDIO DA SILVA, M. 1986a. Estruturação tectônica do Supergrupo Espinhaço na região de Diamantina (MG). Rev. Bras. Geoc., 16(2):212-216.

WEAVER, B.L. \& TARNEY, J. 1981b. The Scourie Dike Suite: petrogenesis and geochemical nature of the Proterozoic subcontinental mantle. Contributions to Mineral, and Petrol. 78:175-188.

WOOD, D.A. 1979. A variable veined suboceanic upper mantle -genetic significance for mid-ocean ridge basalts from geochemical evidence. Geology, 7: 499-503

MANUSCRITO A855

Recebido em 30 de setembro de 1996

Revisão dos autores em 11 de novembro de 1996 Revisão aceita em 12 de novembro de 1996 